\title{
\begin{tabular}{|l|l|l|}
\hline PEDIATRIC & PULMONOLOGY \\
\hline
\end{tabular}
}

\section{Microbiome Characteristics of Induced Sputum compared to Bronchial Fluid and Upper Airway Samples}

\begin{tabular}{|c|c|}
\hline Journal: & Pediatric Pulmonology \\
\hline Manuscript ID & PPUL-17-0455.R2 \\
\hline Wiley - Manuscript type: & Original Article: Respiratory Infections \\
\hline Date Submitted by the Author: & $\mathrm{n} / \mathrm{a}$ \\
\hline Complete List of Authors: & $\begin{array}{l}\text { An, Shi-Qi; Queen's University Belfast Faculty of Medicine Health and Life } \\
\text { Sciences, Wellcome Wolfson Institute for Experimental Medicine } \\
\text { Warris, Adilia; Unviersity of Aberdeen, Institute of Medical Sciences } \\
\text { Turner, Steve; University of Aberdeen, Department of Child Health }\end{array}$ \\
\hline Keywords: & Infections: Pneumonia, TB, Viral \\
\hline Other Keywords: & Microbiota \\
\hline Abstract: & $\begin{array}{l}\text { Objective. The study of the community of microorganisms (the microbiota) } \\
\text { in the lower airways in children is restricted to opportunistic sampling in } \\
\text { children undergoing elective general anaesthetic. Here we tested the } \\
\text { hypothesis that induced sputum is a valid alternative to directly sampling } \\
\text { the lower airways to study lower airway microbiota. } \\
\text { Methods. Children scheduled for elective operations were recruited. Pre- } \\
\text { operatively a sample of induced sputum was obtained. After anaesthesia } \\
\text { was induced, a bronchial brushing and swabs of the upper respiratory tract } \\
\text { were obtained. Bacterial community analysis was performed by } \\
\text { amplification of the V3-V4 } 16 \mathrm{~S} \text { rRNA gene region. } \\
\text { Results. Twenty children were recruited, mean age } 10.7 \text { years. Induced } \\
\text { sputum samples were obtained from } 12 \text { children, bronchial brushing from } \\
14 \text { and nasal, mouth and throat samples in } 15,16 \text { and } 17 \text { children. The } \\
\text { profile of bacterial communities was similar in the mouth, throat and } \\
\text { sputum samples with the nose and bronchial samples being different. } \\
\text { Actinobacteria species dominated the nose and mouth, Fusobacteria were } \\
\text { the dominant species in the throat and sputum whilst Proteobacteria } \\
\text { species dominated in bronchial samples. Forty-one percent of detected } \\
\text { bacteria in bronchial samples were unclassified. Bacterial communities } \\
\text { from the mouth, throat and induced sputum were tightly clustered and } \\
\text { were distinct from nose and those found in bronchial communities. } \\
\text { Conclusions. Induced sputum may not be a valid surrogate for microbiome } \\
\text { assessment of the lower airways in all individuals. Many bacteria in } \\
\text { bronchial samples were not recognised by standard testing, suggesting } \\
\text { that our understanding of the lower airway microbiota in children remains } \\
\text { rudimentary. }\end{array}$ \\
\hline
\end{tabular}


2

3

4

5

6

7

8

9

10

11

12

13

14

15

16

17

18

19

20

21

22

23

24

25

26

27

28

29

30

31

32

33

34

35

36

37

39

40

41

42

43

44

45

46

47

48

49

50

51

52

53

54

55

56

57

58

59

60

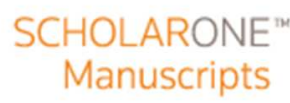

John Wiley \& Sons, Inc. 
Microbiome Characteristics of Induced Sputum compared to Bronchial Fluid and Upper

$$
\text { Airway Samples }
$$

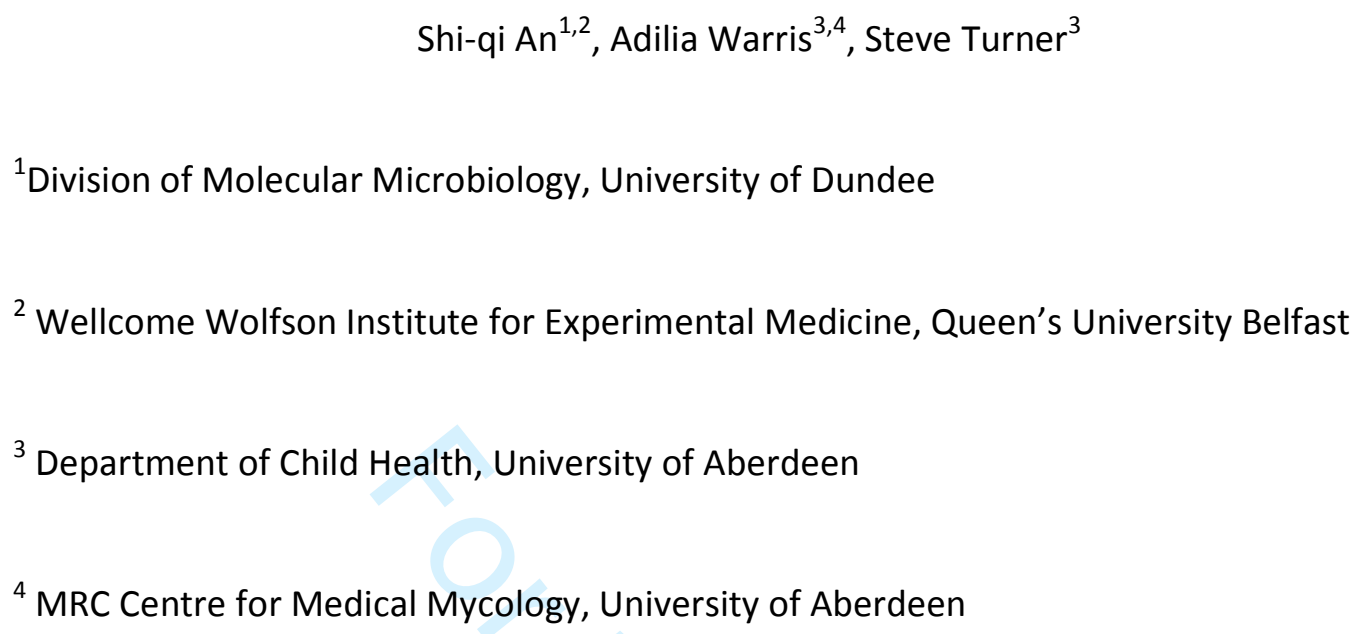

${ }^{1}$ Division of Molecular Microbiology, University of Dundee

${ }^{2}$ Wellcome Wolfson Institute for Experimental Medicine, Queen's University Belfast

${ }^{3}$ Department of Child Health, University of Aberdeen

${ }^{4}$ MRC Centre for Medical Mycology, University of Aberdeen

Contact details: Dr Steve Turner, Child Health, Royal Aberdeen Children's Hospital, Aberdeen, UK, AB25 2ZG. Tel +44 1224 438470. s.w.turner@abdn.ac.uk

Running head: Microbiota of induced sputum

Key words: Bronchial fluid, Child, Microbiota, Sputum 
ABSTRACT

Objective. The study of the community of microorganisms (the microbiota) in the lower airways in children is restricted to opportunistic sampling in children undergoing elective general anaesthetic. Here we tested the hypothesis that induced sputum is a valid alternative to directly sampling the lower airways to study lower airway microbiota.

Methods. Children scheduled for elective operations were recruited. Pre-operatively a sample of induced sputum was obtained. After anaesthesia was induced, a bronchial brushing and swabs of the upper respiratory tract were obtained. Bacterial community analysis was performed by amplification of the V3-V4 16S rRNA gene region.

Results. Twenty children were recruited, mean age 10.7 years. Induced sputum samples were obtained from 12 children, bronchial brushing from 14 and nasal, mouth and throat samples in 15, 16 and 17 children. The profile of bacterial communities was similar in the mouth, throat and sputum samples with the nose and bronchial samples being different. Actinobacteria species dominated the nose and mouth, Fusobacteria were the dominant species in the throat and sputum whilst Proteobacteria species dominated in bronchial samples. Forty-one percent of detected bacteria in bronchial samples were unclassified. Bacterial communities from the mouth, throat and induced sputum were tightly clustered and were distinct from nose and those found in bronchial communities.

Conclusions. Induced sputum may not be a valid surrogate for microbiome assessment of the lower airways in all individuals. Many bacteria in bronchial samples were not recognised by standard testing, suggesting that our understanding of the lower airway microbiota in children remains rudimentary. 


\section{INTRODUCTION}

The lower airways were traditionally considered sterile in healthy individuals, and this paradigm was based on observations that standard microbiology culture from samples collected from healthy individuals yielded no pathogens ${ }^{1}$. The advent of polymerase chain reaction (PCR) testing for bacterial DNA has now identified communities of bacteria in lower airway secretions from healthy individuals ${ }^{2,3}$. The relevance of bacteria in airway microbiota to respiratory symptoms is unclear, but there is evidence suggesting that the microbiota may be relevant to influence respiratory exacerbations $s^{2,4}$ or even to the aetiology of chronic respiratory conditions such as asthma ${ }^{5}$. Exacerbations of chronic conditions such as cystic fibrosis (CF) and chronic obstructive pulmonary disease are linked to a change ${ }^{2}$ (termed "airway dysbiosis") or reduction ${ }^{4}$ in the dominant bacterial species in the microbiota. In young infants, those carrying Haemophilus influenzae or Moraxella catarrhalis in the hypopharynx were more likely to have asthma symptoms at three years of age ${ }^{5}$.

A major challenge to studying the lower airway microbiota is obtaining samples without contamination from the upper airways ${ }^{3}$, and it is known that the microbiota in the naso- and oropharynx differ from that in the bronchus in adults and children ${ }^{6-8}$. Three papers have used bronchoscopic sampling to compare the upper and lower airway microbiome in children and whilst there are similarities in the dominant phyla identified in some studies, there are differences in genera identified and also in the clustering of bacterial communities $^{6-8}$. The characteristics of the lower airway microbiome may also vary between individuals depending on their asthma status ${ }^{6}$ and using a nasal or oral approach to the lower airways ${ }^{8}$. . Two studies in young people with CF have compared the microbiota in 
induced sputum and oropharyngeal swabs ${ }^{9,10}$, and both concluded that a throat swab sample may be a valid surrogate for sputum for microbiota analysis. Induced sputum is a non-invasive alternative to direct bronchial sampling, and is used in the clinical management of tuberculosis and CF in children and also in research. The study of the lower airway microbiome would be considerably easier if induced sputum was a valid surrogate of bronchial fluid. The aim of the current study was to collect induced sputum and samples from the bronchus, nose, throat and mouth in children and undertake a comprehensive assessment of upper and lower airway microbiota in children. Our hypothesis was that induced sputum is a valid alternative to directly sampling the lower airways to study lower airway microbiota.

\section{METHODS}

\section{Study design}

Children aged 5-16 years and scheduled for elective ear nose and throat operations at Royal Aberdeen Children's Hospital between January and June 2015 were invited to take part. All children were scheduled for tonsillectomy and some also had grommets inserted. These operations require endotracheal intubation, which allows sampling of the lower airways without contamination by the upper airways. Children younger than 5 years were excluded since they were unlikely to provide a sample of induced sputum. Other exclusion criteria included receipt of antibiotics within the last month and CF. In the hours before the operation (when the child was fasted), children took part in an assessment which included completion of a questionnaire, height and weight measurement, spirometry (in accordance with international guidelines ${ }^{11}$ ) and induction of sputum using $4 \%$ and if required $5 \%$ saline 
as previously described ${ }^{12}$. An adequate quality sputum sample was defined as the presence of a white sputum plug on a visual inspection; samples were not sufficiently large enough to allow for cell count analysis. Immediately after anaesthesia was induced and the endotracheal tube in situ, a sterile $2.7 \mathrm{~mm}$ interdental brush (Dento Care Professional, London UK) was used to collect a sample of nasal secretions and a separate brush interdental brush was gently brushed on the buccal surface of the mouth to collect oral secretions. A standard bacterial swab (Transwab, Medical Wire Equipment, Corsham, UK) was used to obtain a sample of pharyngeal secretions under direct inspection. Finally, a bronchoscopy cytology brush (10-mm disposable cytology brush, BC 202D-2010, Olympus, Southend-on-Sea, Essex, UK) was passed down the endotracheal tube until resistance was met and then rotated and withdrawn to obtain "blind" bronchial samples. Blood was taken for serum total IgE assay. The study was approved by the North of Scotland Research Ethics Committee (13/NS/0144), written parental consent was obtained and verbal assent was obtained from all participants.

\section{DNA extraction and sequencing}

The bacterial community analysis was carried out as described previously ${ }^{13}$ with some modification as detailed below. Briefly, samples taken from the nose, mouth, throat, sputum and bronchial brushing were frozen at $-80^{\circ} \mathrm{C}$ prior to analysis. Samples were then transported from Aberdeen to Dundee for processing. Upon arrival the sample had thawed and were suspended in phosphate buffer saline where genomic DNA was extracted and purified using the DNA/RNA All Prep kit ${ }^{14}$ (Qiagen) and stored according to manufacturer's instructions. Standard protocol, 16S Metagenomic Sequencing Library Preparation Guide 
(Illumina), was followed to prepare sequencing libraries targeting the variable V3 and V4 regions of the 16S rRNA gene and paired-end sequencing was performed on the MiSeq System (Illumina). We followed sampling and controls procedures described by the Earlham Institute

(http://www.earlham.ac.uk/sites/default/files/docs/Genomic\%20Services/Sample\%20Guide lines\%20Aug17.pdf) and Illumina

(https://support.illumina.com/documents/documentation/chemistry documentation/16s/1 6s-metagenomic-library-prep-guide-15044223-b.pdf

which are designed to minimize the risk for contamination. Quality controls and sequencing were performed at Earlham Institute (Norwich, UK).

\section{Data and Statistical analysis}

Bioinformatics analyses on raw data was performed using the Biomedical Genomics Workbench version 4.0 (Qiagen) equipped with the Microbial Genomics Module version 2.0 (Qiagen) plugin. Sequences were imported and processed for optional merge paired reads, adapter trimming, fixed length trimming and then the sequences were filtered based on the number of reads to obtain sequences that are comparable in length and coverage for clustering. Quality and chimera filtering were performed using the recommended programme parameters [for complete details see www.qiagenbioinformatics.com]. Samples with low coverage were removed from further analysis. Operational Taxonomic Units (OTU) clustering and taxonomic assignment were done using Greengenes v13_5 (97\%) as reference. New OTUs were indicated when similarity percentage was lower than $80 \%$ with minimum occurrence of 5 reads. Low abundant OTUs were discarded from further analyses (minimum combined abundance was set at 10). Summary of processed sequence data is 
described in Table S1. MUSCLE was used for OTUs alignment in order to reconstruct a maximum likelihood phylogeny with neighbor joining as construction method and Jukes Cantor as nucleotide substitution model. We compared community structures and diversity across patient cohorts to determine if inter-cohort differences in structure were seen. To achieve this a rarefaction sampling analysis was carried out using a standard methodology ${ }^{15}$. In order to minimise the risk for contamination, we sought to identify a high (i.e. conservative) number of sequences required to characterise bacteria from the samples. In the absence of a standard international stringency cut off the default settings in the workbench software (Qiagen) were applied; the default settings filters out many reads and generates fewer unassigned reads and is therefore highly stringent. These adjustments were assessed by analyzing a previously characterized mock community to confirm that the data handling was consistent with other studies (sequencing files of the project: ERP021973,https://www.ebi.ac.uk/metagenomics/projects/ERP021973/samples/ERS15889

32). Data generated in the study was assessed using the Qiagen software and also applied to EBI Metagenomics analysis pipeline V3.0 to verify results (acknowledging that fewer reads would remain in the analysis with Qiagen). The findings in the controls were taken into account during the analysis. Alpha diversity was calculated using number of OTUs. Beta diversity was obtained using D_0.5 UniFrac and represented as Principal Coordinate Analysis (PCOA). Robustness analysis was performed using PERMANOVA with UniFrac distances. All sequencing data have been deposited in NCBI Sequence Read Archive (SRA) database under the follow BioProject ID: PRJNA388557 


\section{RESULTS}

\section{Study Subjects}

Twenty patients recruited of which 8 were boys, 7 with diagnosed asthma and the mean (standard deviation) age was 10.7 (2.8) years. Descriptives of the children recruited are given in Table 1. Induced sputum was obtained from 12 children and samples of fluid from bronchus, throat mouth and nose were obtained in all children. Three control samples were collected.

\section{Sample quality control}

A total of $11,750,879$ PCR reads were were analysed, and 606,227 high quality reads were obtained. The resulting OTU table contained 1053 OTUs, where 532 OTUs were assigned based on the Greengenes v13_5 (clustered at 97\% similarity), and 521 OTUs were novel (see Table S1). These processing criteria allowed data from 74 samples from patients for further analyses. Of these 15 were from the nose, 16 from mouth, 17 from throat, 12 from sputum and 14 from bronchial samples (Table S2). Samples from all five sites were available in six children. The median read counts for the five sites were as follows: bronchus 4438; mouth 4400; throat 4868; nose 8927 ; and sputum 11250 . The results from the mock community analysis using Qiagen and EBI Metagenomics analysis pipelines were highly consistent. However Qiagen software assigned phyla for all samples whereas the EBI Metagenomics did not assign phyla in $15 \%$ of samples (see Table S3).

\section{Bacterial abundance}


The relative bacterial abundance in each sample cohort is presented in Figure 1. The overall profile of bacterial communities was largely similar in the mouth, throat and sputum samples with the nose and bronchial being different. All sample cohorts contained five major bacterial phyla: Actinobacteria, Bacteroidetes, Firmicutes, Fusobacteria and Proteobacteria. The three most abundant phyla of bacteria observed by analysis in nose, mouth, throat, bronchial and sputum were Actinobacteria, Fusobacteria and Bacteroidetes (seen in Figure 1). Actinobacteria dominated in the nose (73\% of all total species) and mouth (37\%) samples, Fusobacteria dominated in throat (31\%) and sputum (38\%) samples and Proteobacteria were dominant in bronchial samples (34\%). Both nasal and bronchial samples had more unclassified OTUs ( $12 \%$ and $41 \%$ respectively) than other samples. The relative abundance of each individual sample is shown in Figure 2. Figure S1 presents the phylogenetic tree of the microbial community in all analysed samples. In two of the control samples there was no identifiable bacteria DNA signal however some DNA signal was detected in the one of the three control and this was taken into account during analysis (See Figure 2).

\section{Comparison between complete community structures within samples from different sites}

The rarefaction analysis suggested that 2000 sequences would be sufficient for characterizing the majority of bacteria present in these communities. The numbers of reads were lowest in bacterial communities from mouth and bronchus (Figure 3; Table S2). A comparison of individual bacterial communities, using PERMANOVA principal coordinate analysis (PCOA), showed a distinct clustering by sample cohorts based roughly on where the sample was taken from. Bacterial communities in samples from the mouth, throat and induced sputum were tightly clustered together $(\operatorname{padj}=1)$ and were distinct from those of 
the nose and bronchus (padj<0.001), which independently clustered (Figure 4; Table 2); these clustering results were seen when children with asthma and without asthma were considered separately (Table S4), Table S5 demonstrates that clustering of bacterial communities was not evident between sites among subgroups stratified by asthma/not asthma or atopy/not atopy.

The bacterial communities in the five sites sampled did not differ between the children with and without asthma; there was evidence of some differences in bacterial abundance and diversity between groups (see supplement Figure S2). A comparison of the abundant phyla of bacteria identified in bronchial samples taken from asthma and non-asthma patients showed the profiles were similar, but the abundance of a signal for phyla was different (but did not reach significance). In bronchial samples from asthma patients, the abundant phyla identified was Proteobacteria while those samples taken from non-asthma patients showed a greater abundance for Fusobacteria.

\section{DISCUSSION}

This study was designed to determine whether induced sputum is a valid alternative to directly sampling of the lower airways to study airway microbiota in children. The main finding was that the characteristics of the microbiota in induced sputum and in bronchial samples were different. A second finding was that a minority of bacterial DNA in bronchial samples was unclassified. In two individuals (P12 and P16, see Figure 2) the proportion of phyla identified from the bronchial sample was similar to the sputum sample but for the remainder of participants there were clear differences in the proportions of different phyla 
from bronchial and sputum samples. Together these findings suggest that induced sputum is not a valid surrogate for direct bronchial sampling to study the lower airway microbiota in all children, but that there is still a pressing need to better understand the lower airway microbiota in children. Opportunistic bronchial sampling under clinically-indicated general anaesthetic should remain the gold standard for studying lower airway microbiome in children.

To our knowledge there are only three studies which have compared the microbiota of upper and lower airway secretions in healthy children or children with asthma ${ }^{6-8}$. A study where the majority of recruits were adults showed that the bacterial community in the oropharynx and bronchus were similar in healthy individuals ${ }^{6}$. One solely paediatric study found very little overlap between the microbiota of the oropharynx and lower airways ${ }^{7}$ whilst a second paediatric study found similar predominant phyla in upper and lower airway samples but different genera ${ }^{8}$. Both paediatric studies found significant differences in the clustering of bacterial communities in the upper and lower airways ${ }^{7,8}$. The dominant phyla in bronchial samples is not consistent between studies but there is consistency for upper airway samples. For example, the microbiota of the upper airways is predominated by bacteria from the Firmicutes (e.g. Staphylcoccus and Streptococcus) and the Actinobacteria phyla (e.g. Corynebacteria) in our study and three others ${ }^{6-8}$. In contrast and in bronchial samples, bacterial species within the Proteobacteria phylum (including Moraxella and Haemophilus) predominated in our study and one other ${ }^{7}$ but Firmicutes dominated in a third study ${ }^{8}$., Differences in lower airway microbiome between studies may be due to different sampling methods ${ }^{8}$ and treatment with current or recent antibiotics ${ }^{7,8}$. Our results, where we find no differences between the microbiome of induced sputum and throat swab, 
are also consistent with previous studies which suggested that a throat swab may be a useful surrogate of induced sputum in young children with $\mathrm{CF}^{9,10}$. As our study did not include children with CF we cannot comment on the validity of induced sputum as an index of lower airway infection in this specific clinical setting. The consistency of our results with previous studies assures us that our findings are valid, despite the relatively small sample size.

Our study findings were based on samples taken from a group of children who were well. Recent work has given insight into the relationship between airway microbiota in the upper airways of children with acute infection, with results which differ from the present study. For example there is evidence that in the context of acute upper and lower respiratory tract infection, different bacterial species predominate in the nasopharynx (e.g. Proteobacteria and Firmicutes which contrast with Actinobacteria and Fusobacteria in our study ${ }^{15}$. The characteristics of the nasopharyngeal microbiota also change during the course of acute otitis media infection ${ }^{16}$.

Our study design minimised the risk of false positive results arising from contamination and from samples containing low DNA yields. There is no consensus on the number of control samples which should be collected for microbiome studies, and two of the three controls we collected contained no bacterial DNA and a third contained only bacteria with a very different profile of phyla compared to the samples from study subjects. We set out to have a high threshold for reads (>2000) and this filtered out samples with low DNA yields. Another source of potential contamination comes from reagents used to analyse the samples and this risk was minimised by taking standard precautions were taken and by including control samples in the analysis. Whilst we cannot exclude the possibility that 
some contamination may have occurred, our robust methodology and the consistency of our findings with previous studies assure us that any contamination has not substantially affected our findings.

Finding a small proportion of unassigned bacterial DNA in the nose and bronchial samples was unexpected and worthy of further investigation. The presence of "unclassified bacteria" has been described in nasopharyngeal samples from children ${ }^{7}$, but not in bronchial samples. We have carefully reviewed the unassigned sequences and removed human DNA sequences, and we therefore believe that there are a number of bacteria in the respiratory tract which are not identified by standard microbiota methodologies currently used. We analysed data from a mock community (a "positive control") and this demonstrated that the Qiagen software used for analysis of our samples was able to detect bacteria in similar proportions to another software "pipeline". The Qiagen software identified all phyla whereas the alternative software could not assign $15 \%$ of bacterial DNA and this may be explained by the higher stringency set for the Qiagen software. There is not standard for stringency but these results suggest that although the higher stringency reduces the number of reads included in the analysis, the results are highly comparable to other methods and possibly less affected by potential contaminants. Our data are publicly available and colleagues are welcome to apply different stringencies to our data.

There are a number of settings where upper airway samples have been shown to be valid surrogates of lower airway samples for the clinical diagnosis of infective and non-infective conditions. Viral aetiology in bronchiolitis (a lower respiratory tract infection) can be established by nasopharyngeal aspirate ${ }^{16}$ and the nasal mucosa is used for diagnosis of primary ciliary dyskinesia ${ }^{17}$ and (in some situations) $\mathrm{CF}^{18}$. In the research setting, sampling 
from the upper airways has been validated as a surrogate for lower airway sampling ${ }^{19,20}$. However, we demonstrated that the microbiota of the nose was distinct to mouth and bronchus in most individuals and our results indicating that the microbiota of the upper airway is probably not a valid surrogate of lower airway microbiota in children. Previous papers have suggested that with regards to lower airway samples, upper airway samples are “imperfect but reliable"7 and "both similar and different" ${ }^{8}$ and overall, our work finds more evidence for the upper airways samples being "imperfect and different" to lower airway samples and not "reliable and similar". .

The novelty of our study is that we demonstrate how the microbiota of induced sputum differs from bronchial samples and very closely matches the microbiota of mouth and throat, most likely due to contamination of lower airway secretions as they pass through the oropharynx, including sputum which is commonly mixed with expectorant. Our findings are consistent with a study of 78 children (mean age 2 years, almost half having received recent antibiotic treatment) which also describes differences in the microbiota between nose and mouth and between mouth and bronchial fluid ${ }^{7}$. Consistent with our findings, a study of adults and children (mean age 11 years) reports Actinobacteria being the predominant phyla in the nose but being almost absent from the oropharynx and bronchus. A different pattern is shown for Proteobacteria which are increasing from nose through to oropharynx and bronchus ${ }^{6}$. The apparent presence of different dominant bacterial communities in the upper and lower airways raises the question "where do bacteria in the lower airways originate?"

In children the lower airways are in direct communication with and adjacent to the oropharynx but there are several mechanisms that may lead to the establishment of 
different bacterial communities above and below the vocal cords. Perhaps most obviously, a child's mouth is regularly exposed to food and inedible items introduced to the oral cavity (including cutlery and fingers) whereas the lower airways are exposed only to inhaled exposures. Micro aspiration due to laryngopharyngeal reflux is thought to occur on a regular basis ${ }^{21}$ and exposure to acid and other gastric contents (including bacteria) may affect the microbiota of the lower airways but not the oropharynx. There are differences in the innate defences of the upper and lower airways which may also explain differences in their respective microbiota, for example lactoferrin concentrations are twice as high in the lower airways relative to upper airways ${ }^{22}$ whereas nitric oxide concentrations (known to have antimicrobial properties ${ }^{23}$ ) are typically one hundred times higher in the upper airways.

Our study was not designed to relate microbiota to clinical phenotypic data collected, for example asthma or atopy. However, in light of recent studies examining these parameters in children with asthma ${ }^{6,7}$ we made the best use of the data available, even with the limited number we had at our disposal. When examined the possible relationship between the bacteria present in children with and without asthma or atopy, we found no statistically differences between the bacteria when the samples were examined as a whole group or as specific sample region cohorts (nose, mouth, throat, bronchial and sputum samples), Figure S2 and Table S3. A descriptive analysis of the bronchial samples collected found Protoebacteria to be the dominant species and this is consistent with Hilty et al ${ }^{6}$. 
There are some limitations to our study. First, the number of participants was relatively small. Second, induced sputum was not obtained in all participants and not all samples met our quality control criteria for bacterial DNA analysis and this resulted in missing data for some individuals. Third, as has been reported in other sputum microbiome studies ${ }^{6,7}$, we were not able to perform cell counts on the sputum pellet to determine the presence of neutrophils and absence of squamous epithelial cells. Finally, we did not prospectively calculate the DNA yield from samples although the consistency of our results with the small number of published studies ${ }^{6,7}$, the consistency of results using both the Qiagen and EBI Metagenomics pipelines and the comparable results from mock communities assure is that Iow DNA yield has not altered the results.

In summary, we report that neither induced sputum nor swabs from nose or mouth give an accurate indication of the bronchial microbiota in all children. For very obvious practical and ethical reasons, upper airway sampling will be necessary to study airway microbiome in many research setting and our findings adds to the evidence describing the limitations of this pragmatic approach. Our study was not designed to compare differences in the lower airway microbiota of children with and without asthma, and future research is required to supplement the little we do know about this potentially important subject.

\section{ACKNOWLEDGEMENTS}

The authors would like to thank the study participants and their parents. The study was supported by an NHS-Grampian Endowments. AW is supported by the Wellcome Trust 
Strategic Award (grant 097377) and the MRC Centre for Medical Mycology (grant MR/N006364/1) at the University of Aberdeen.

Competing interest statement. None of the authors has any competing interests.

\section{REFERENCES}

1. Kahn FW, Jones JM. Diagnosing bacterial respiratory infection by bronchoalveolar lavage. J Infect Dis 1987; 155(5): 862-9.

2. Marsland BJ, Yadava K, Nicod LP. The airway microbiome and disease. Chest 2013; 144(2): 632-7.

3. Rogers GB, Shaw D, Marsh RL, Carroll MP, Serisier DJ, Bruce KD. Respiratory microbiota: addressing clinical questions, informing clinical practice. Thorax 2015; 70(1): 74-81.

4. Klepac-Ceraj V, Lemon KP, Martin TR, Allgier M, Kembel SW, Knapp AA, Lory S, Brodie EL, Lynch SV, et al. Relationship between cystic fibrosis respiratory tract bacterial communities and age, genotype, antibiotics and Pseudomonas aeruginosa. Environ Microbiol 2010; 12(5): 1293-303.

5. Bisgaard H, Hermansen MN, Bonnelykke K, Stokholm J, Baty F, Skytt NL, Aniscenko J, Kebadze T, Johnston SL. Association of bacteria and viruses with wheezy episodes in young children: prospective birth cohort study. BMJ 2010; 341: 4978.

6. Hilty M, Burke C, Pedro H, Cardenas P, Bush A, Bossley C, Davies J, Ervine A, Poulter L, Pachter L, et al. Disordered microbial communities in asthmatic airways. PLOS ONE 2010; 5(1): e8578. 
7. Marsh RL, Kaestli M, Chang AB, Binks MJ, Pope CE, Hoffman LR, Smith-Vaughan HC. The microbiota in bronchoalveolar lavage from young children with chronic lung disease includes taxa present in both the oropharynx and nasopharynx. Microbiome 2016; 4: 37.

8. Kloepfer KM, Deschamp AR, Ross SE, Peterson-Carmichale SL, Hemmerich CM, Rusch DB, Davies SD. In children, the microbiota of the nasopharynx and bronchoalveolar lavage fluid are both similar and different. Ped Pulmonol 2018; DOI: 10.1002/ppul.23953

9. Zemanick ET, Wagner BD, Robertson CE, Stevens MG, Szefler SJ, Accurso FJ, Sagel SD, Harris JK. Assessment of airway microbiota and inflammation in cystic fibrosis using multiple sampling methods. Ann Am Thor Soc 2015; 12(2): 221-9.

10. Boutin S, Graeber SY, Weitnauer M, Panitz J, Stahl M, Clausznitzer D, Kaderali L, Einarsson G, Tunney MM, Elborn S, et al. Comparison of microbiomes from different niches of upper and lower airways in children and adolescents with cystic fibrosis. PLOS ONE 2015; 10(1): e0116029.

11. Miller MR, Hankinson J, Brusasco V, Burgos F, Casaburi R, Coates A, Crapo R, Enright P, van der Grinten CP, Gustaffson P, et al. Standardisation of spirometry. Eur Respir J 2005; 26(2): 319-38.

12. Pontin J, Blaylock MG, Walsh GM, Turner SW. Sputum eosinophil apoptotic rate is positively correlated to exhaled nitric oxide in children. Pediatr Pulmonol 2008; 43(11): 1130-4. 
13. Twomey KB, Alston M, An S, O'Connell OJ, McCarthy Y, Swarbreck D, Febrer M, Dow JW, Plant BJ, Ryan RP. Microbiota and metabolite profiling reveal specific alterations in bacterial community structure and environment in the cystic fibrosis airway during exacerbation. PLOS ONE 2013; 8(12): e82432.

14. Moen AEF, Tannaes TM, Vatn S, Ricanek P, Vatn MH, Jahnsen J, IBD-Character Consortium. Simultaneous purification of DNA and RNA from microbiota in a single colonic mucosal biopsy. BMC Research Notes 2016; 9: 328.

15. Zakharkina T, Martin-Loeches I, Matamoros S, Povoa P, Torres A, Kastelijn JB, Hofstra JJ, de Wever B, de Jong M, Schultz MJ, et al. The dynamics of the pulmonary microbiome during mechanical ventilation in the intensive care unit and the association with occurrence of pneumonia. Thorax 2017; 72: 803-10.

16. Kelly MS, Surette MG, Smieja M, Pernica JM, Rossi L, Lunistra, Steenhoff AP, Feemster KA, Goldfarb DM, Arscott-Mills T, Bioditswe S, Rulaganyang I et al. The Nasopharyngeal Microbiota of Children with Respiratory Infections in Botswana. Pediatr infect Dis J 2017;36:e211-e218

17. Chonmaitree T, Jennings K, Golovko G, Khanipov K, Pimenova M, Patel JA, McCormick DP, Loeffelholz MJ, Fofanov, Y. Nasopharyngeal microbiota in infants and changes during viral upper respiratory tract infection and acute otitis media. PLoS ONE 2017;12 (7):e0170630. 
18. Ralston SL, Lieberthal AS, Meissner HC. Ralston SL, Lieberthal AS, Meissner HC, Alverson BK, Baley JE, Gadomski AM, Johnson DW, et al. Clinical Practice Guideline: The Diagnosis, Management, and Prevention of Bronchiolitis. Pediatrics. 2014;134(5):e1474e1502. Pediatrics 2015; 136(4): 782.

19. Barbato A, Frischer T, Kuehni CE, Snijders D, Azevedo I, Bakati G, Bartoloni L, Escribano A, Haarman E, Messelmar B, et al. Primary ciliary dyskinesia: a consensus statement on diagnostic and treatment approaches in children. Eur Respir J 2009; 34(6): 1264-76.

20. Farrell PM, Rosenstein BJ, White TB, Accurso FJ, Castellani C, Cutting GR, Durie PR, Legrys VA, Massie J, Parad RB, et al. Guidelines for diagnosis of cystic fibrosis in newborns through older adults: Cystic Fibrosis Foundation consensus report. J Pediatr 2008; 153(2): S4-S14.

21. Pringle EJ, Richardson HB, Miller D, Cornish DS, Devereux GS, Walsh GM, Turner SW. Nasal and bronchial airway epithelial cell mediator release in children. Pediatr Pulmonol 2012; 47(12): 1215-25.

22. Pitrez PMC, Brennan S, Turner S, Sly PD. Nasal wash as an alternative to bronchoalveolar lavage in detecting early pulmonary inflammation in children with cystic fibrosis. Respirology 2005; 10(2): 177-82.

23. Venkatesan NN, Pine HS, Underbrink M. Laryngopharyngeal reflux disease in children. Pediatr Clin North Am 2013; 60(4): 865-78. 
24. Roca-Ferrer J, Mullol J, Perez M, Xaubet A, Momlins L, de Haro J, Shelhamer J, Picado C. Effects of topical glucocorticoids on in vitro lactoferrin glandular secretion: comparison between human upper and lower airways. J Allergy Clin Immunol 2000; 106(6): 1053-62.

24. Fang FC. Antimicrobial reactive oxygen and nitrogen species: concepts and controversies. Nature Rev Microbiol 2004; 2(10): 820-32. 


\section{FIGURE LEGENDS}

Figure 1. Relative abundances of bacterial phyla identified as operational taxonomic units (OTUs) from the sequence reads generated from airway samples taken from children. The bar chart illustrates the taxonomic composition of each cohort of samples from a particular site. A detailed summary of the bacteria in each sample is described in Figure 2.

Figure 2. Operational Taxonomic Units based relative sequence abundance of bacterial phyla based on $16 \mathrm{~S}$ rRNA of all 75 samples. A detailed summary of the bacteria in each sample is described. On the horizontal axis, the number following $\mathrm{P}$ is the patient number and $\mathrm{NO}=$ nose, $\mathrm{MO}=$ mouth, $\mathrm{TH}=$ throat, $\mathrm{SP}=$ Sputum, $\mathrm{BR}=$ bronchial sample. $\mathrm{C} 2=$ the control sample where bacterial DNA was detected. $N / A=$ not applicable

Figure 3. Alpha diversity rarefaction curves of samples based on total number of observed Operational Taxonomic Units. Colour denotes different sample cohort ("nose" (NO), "mouth" (MO), "throat" (TH), "sputum" (SP) and "bronchial" (BR) samples).

Figure 4. Diversity analysis demonstrating differences in the bacterial phyla community between samples taken from airway samples taken from asthmatic and non-asthmatic children. Principal coordinate (PCo) analysis of all samples based on D_0.5 UniFrac distance. Colour denotes different sample cohort ("nose" (NO), "mouth" (MO), "throat" (TH), "sputum" (SP) and "bronchial" (BR) samples). Y=sample from child with asthma.

Figure S1. Phylogenetic tree of the microbial community in all analysed samples. All Operational Taxonomic Units are shown in this figure.

Figure S2. Bacterial phyla abundance and diversity between groups. On the horizontal axis, the first letter " $\mathrm{Y}$ " indicates that the child had asthma and " $\mathrm{N}$ " that they did not have 
asthma and $\mathrm{NO}=$ nose, $\mathrm{MO}=$ mouth, $\mathrm{TH}=$ throat, $\mathrm{SP}=\mathrm{Sputum}, \mathrm{BR}=$ bronchial sample. $\mathrm{N} / \mathrm{A}=$ not applicable

\section{TABLE LEGENDS}

Table 1. Details of the children recruited. SABA=short acting beta agonist, ICS=inhaled corticosteroids, LABA=long acting beta agonist

Table 2. PERMANOVA analysis results of testing differences in beta-diversity among different sample sites.

Table S1. Summary of sequence data used for Operational Taxonomic Units (OTU) clustering and analysis. The number of filtered reads and reads in OTUs doee not include the reads excluded by quality control

Table S2. List of sequence data for individual samples used for Operational Taxonomic Units (OTU) clustering and analysis.

Table S3. Comparison of results from two pipelines used to analyse data. The Qiagen pipeline was used for the present study. The EBA pipeline (full name EBI Metagenomics analysis pipeline V3.0) was used as a comparator

Table S4. PERMANOVA analysis separately comparing beta-diversity among different sample sets for children with asthma and without asthma.

Table S5. PERMANOVA analysis comparing beta-diversity among different sample sets. Atopy was defined as serum IgE $>100 \mathrm{kU} / \mathrm{l}$. 


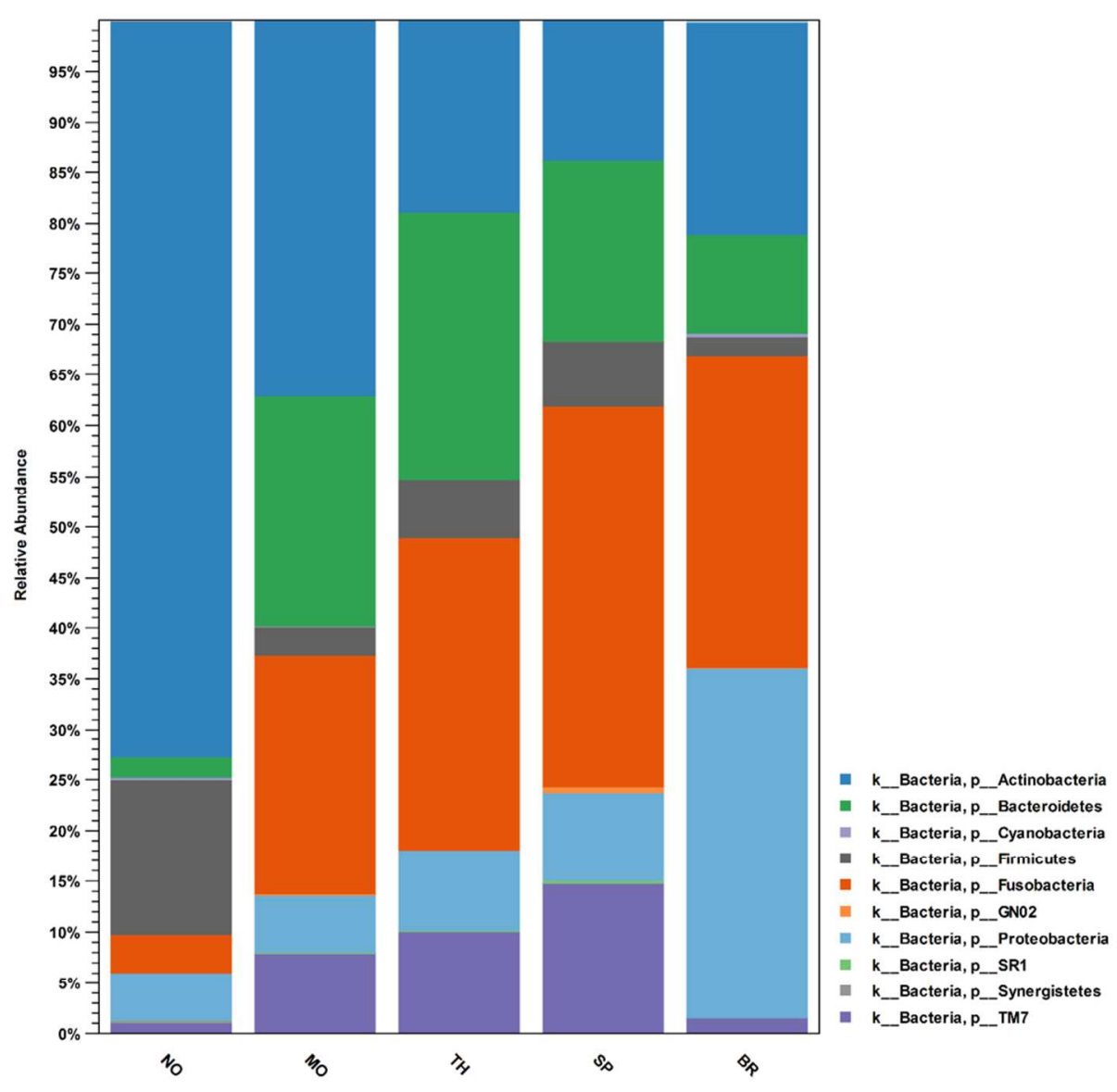

Figure 1. Relative abundances of bacterial phyla identified as operational taxonomic units (OTUs) from the sequence reads generated from airway samples taken from children. The bar chart illustrates the taxonomic composition of each cohort of samples from a particular site. A detailed summary of the bacteria in each sample is described in Figure 2.

$193 \times 175 \mathrm{~mm}(150 \times 150 \mathrm{DPI})$ 
Figure 2. Operational Taxonomic Units based relative sequence abundance of bacterial phyla based on $16 \mathrm{~S}$ rRNA of all 75 samples. A detailed summary of the bacteria in each sample is described. On the horizontal axis, the number following $\mathrm{P}$ is the patient number and $\mathrm{NO}=$ nose, $\mathrm{MO}=$ mouth, $\mathrm{TH}=$ throat, $\mathrm{SP}=\mathrm{Sputum}$, $\mathrm{BR}=$ bronchial sample. $\mathrm{C} 2=$ the control sample where bacterial DNA was detected. $\mathrm{N} / \mathrm{A}=$ not applicable

$338 \times 190 \mathrm{~mm}(96 \times 96 \mathrm{DPI})$ 


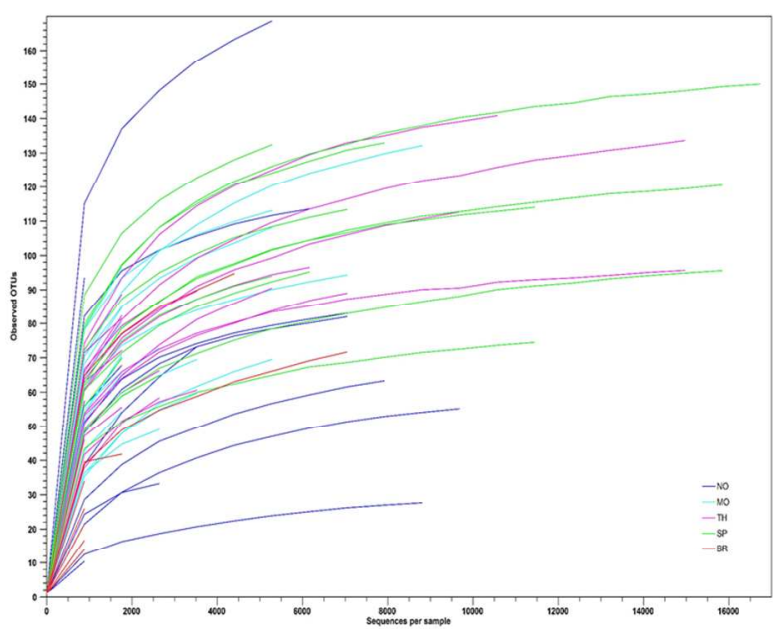

Figure 3. Alpha diversity rarefaction curves of samples based on total number of observed Operational Taxonomic Units. Colour denotes different sample cohort ("nose" (NO), "mouth" (MO), "throat" (TH), "sputum" (SP) and "bronchial" (BR) samples).

$338 \times 190 \mathrm{~mm}(96 \times 96 \mathrm{DPI})$ 
Figure 4. Diversity analysis demonstrating differences in the bacterial phyla community between samples taken from airway samples taken from asthmatic and non-asthmatic children. Principal coordinate (PCo) analysis of all samples based on D_0.5 UniFrac distance. Colour denotes different sample cohort ("nose" (NO), "mouth" (MO), "throat" (TH), "sputum" (SP) and "bronchial" (BR) samples). Y=sample from child with asthma.

\section{$254 \times 190 \mathrm{~mm}(96 \times 96 \mathrm{DPI})$}


Table 1. Details of the children recruited. SABA=short acting beta agonist, ICS=inhaled corticosteroids, LABA=long acting beta agonist

\begin{tabular}{|c|c|c|c|c|}
\hline & & $\begin{array}{c}\text { All children } \\
(n=20 \text { unless stated) }\end{array}$ & $\begin{array}{l}\text { Children with asthma } \\
\text { (n=7 unless stated) }\end{array}$ & $\begin{array}{c}\text { Children without } \\
\text { asthma ( } \mathrm{n}=13 \text { unless } \\
\text { stated) }\end{array}$ \\
\hline \multicolumn{2}{|r|}{ Mean age (SD), y } & $10.7(2.8)$ & $12.1(2.2)$ & $9.9(2.9)$ \\
\hline \multicolumn{2}{|c|}{ Proportion male (number) } & $40 \%(8)$ & $43 \%(3)$ & $39 \%(5)$ \\
\hline \multicolumn{2}{|c|}{ Mean $\% \mathrm{FEV}_{1} / \mathrm{FVC}$ ratio (SD) } & $103 \%(8) n=19$ & $101 \%(9.5)$ & $104 \%(8) n=12$ \\
\hline \multicolumn{2}{|c|}{ Median plasma IgE (SEM), kU/I } & $118(36) n=19$ & $193(48) n=6$ & $82(49)$ \\
\hline \multicolumn{2}{|c|}{ Mean BMI centile (SD) } & $70 \%(28)$ & $64 \%(28)$ & $73 \%(28)$ \\
\hline \multirow{2}{*}{$\begin{array}{l}\text { Proportion } \\
\text { where the }\end{array}$} & Bronchial fluid & $70 \%(14)$ & $86 \%(6)$ & $62 \%(8)$ \\
\hline & Induced & $60 \%(12)$ & $86 \%(6)$ & $46 \%(6)$ \\
\hline sample met the & sputum & & & \\
\hline quality control & Throat swab & $85 \%(17)$ & $100 \%$ & $77 \%(10)$ \\
\hline \multirow{2}{*}{$\begin{array}{l}\text { criteria for } \\
\text { analysis (n) }\end{array}$} & Mouth swab & $80 \%(16)$ & $100 \%$ & $69 \%(9)$ \\
\hline & Nose swab & $75 \%(15)$ & $100 \%(7)$ & $62 \%(8)$ \\
\hline \multicolumn{2}{|c|}{ Asthma treatment } & & $\begin{array}{c}2 \text { SABA only } \\
2 \text { ICS plus SABA } \\
3 \text { ICS, LABA plus SABA }\end{array}$ & \\
\hline
\end{tabular}


Table 2. PERMANOVA analysis results of testing differences in beta-diversity among

\begin{tabular}{|c|c|c|c|c|c|}
\hline Sample site 1 & Sample site 2 & $\begin{array}{c}\text { Number of } \\
\text { individuals with } \\
\text { comparable } \\
\text { data }\end{array}$ & $\begin{array}{l}\frac{\text { Number of }}{\text { samples included }} \\
\frac{\text { in the analysis }}{\text { (site } 1 \& \text { site } 2)}\end{array}$ & $\begin{array}{c}\text { p value } \\
\text { (unadjusted) }\end{array}$ & $\begin{array}{c}\text { p value } \\
\text { (adjusted for } \\
\text { multiple testing) }\end{array}$ \\
\hline Nose & Mouth & 11 & $15 \& 16$ & 0.00001 & 0.00015 \\
\hline Nose & Throat & 13 & $15 \& 17$ & 0.00001 & 0.00015 \\
\hline Mouth & Throat & 14 & $16 \& 17$ & 0.22171 & 1 \\
\hline Nose & Sputum & 10 & $15 \& 11$ & 0.00001 & 0.00015 \\
\hline Mouth & Sputum & 10 & $16 \& 11$ & 0.17806 & 1 \\
\hline Throat & Sputum & 10 & $17 \& 11$ & 0.27851 & 1 \\
\hline Nose & Bronchus & 12 & $15 \& 14$ & 0.00002 & 0.0003 \\
\hline Mouth & Bronchus & 9 & $16 \& 14$ & 0.00001 & 0.00015 \\
\hline Throat & Bronchus & 12 & $17 \& 14$ & 0.00001 & 0.00015 \\
\hline Sputum & Bronchus & 10 & $11 \& 14$ & 0.00003 & 0.00045 \\
\hline
\end{tabular}




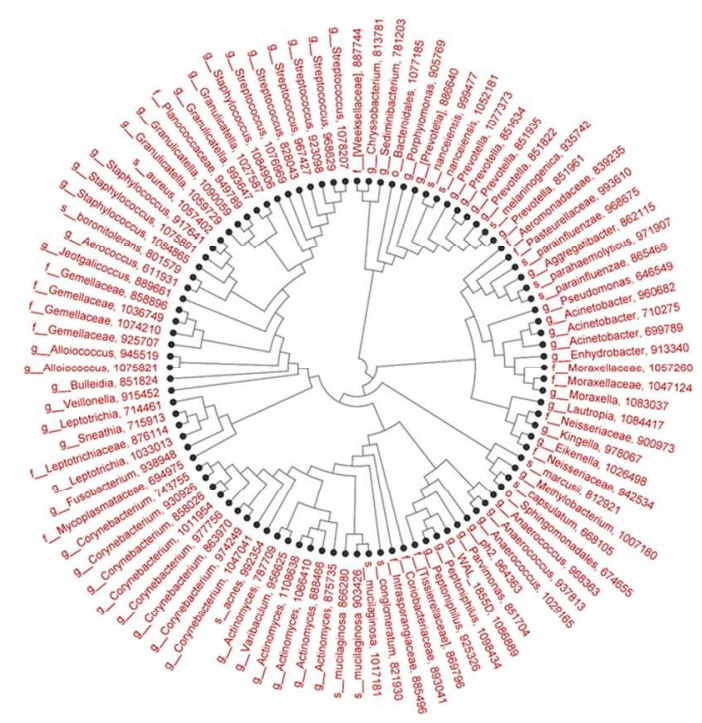

$338 \times 190 \mathrm{~mm}(96 \times 96 \mathrm{DPI})$ 


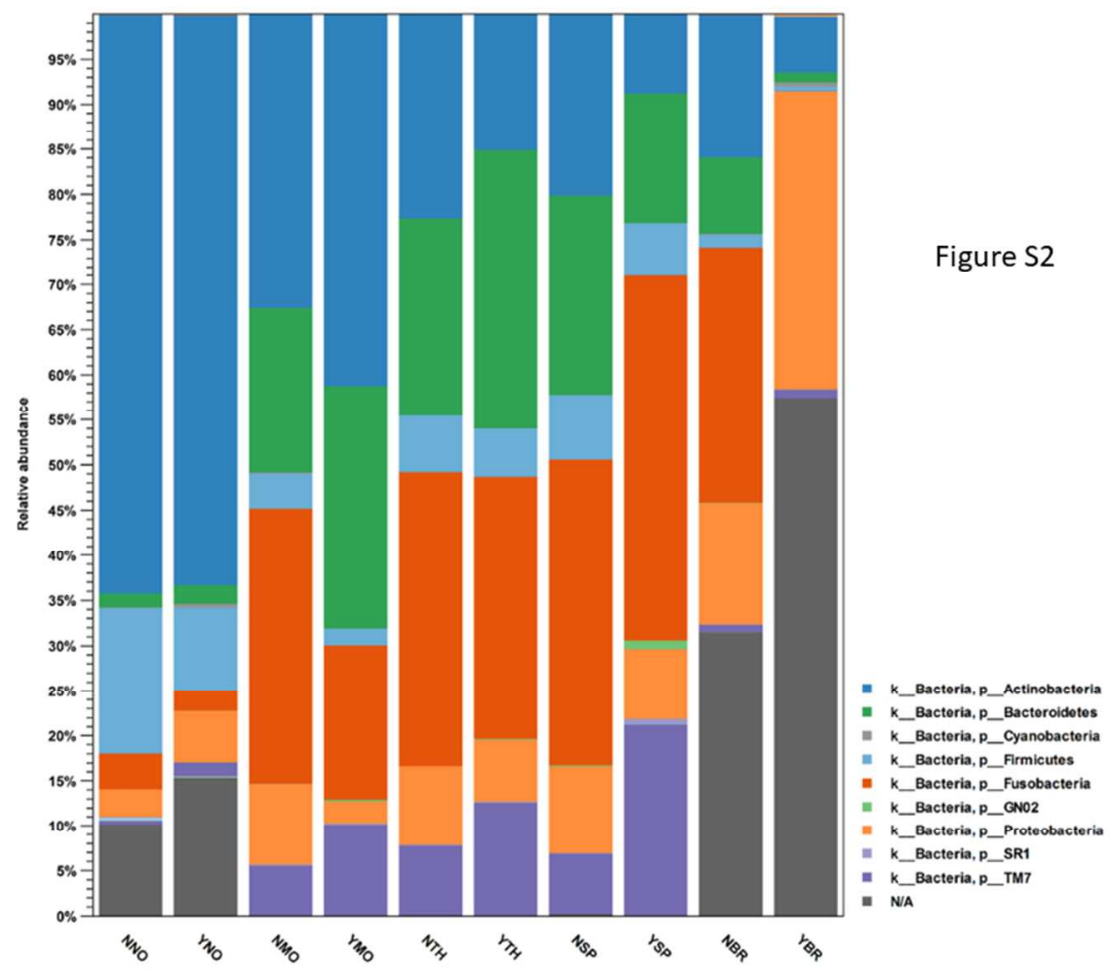

$254 \times 190 \mathrm{~mm}(96 \times 96 \mathrm{DPI})$

John Wiley \& Sons, Inc. 
Whereas some minor issues have been addressed major issues are still not sufficiently replied to. I am still not convinced that one the main messages, namely that bronchial samples differ significantly from induced sputum, is justified by the data and therefore allows to conclude: "Induced sputum may not be a valid surrogate for microbiome assessment of the lower airways."

Before going into a deeper analysis I would like to mention that a considerable part of confusion comes due to erroneous labeling and numbering of the figures and tables. E.g. in my previous review I referred in point 4 to Fig. S2 which according to the legends was the phylogenetic tree (yet the Figure itself had no label) and claimed the absence of Streptococci and Haemophilus. In the reply the authors now refer to bar graphs that of course only show phyla. This is not a reply to my question. Moreover the current manuscript is still superficial in preparation: Fig. S2 as labeled in the legends ("Figure S2. Bacterial phyla abundance and diversity between groups") is sjown as Fig. S3 on p.30. In Fig. 1 the legend gives a note "A detailed summary of the bacteria in each sample is described in Figure S1." but this is now Fig. 2. Therefore I now always refer to the page number indicated at the top of the pdf file.

Our response: We apologise for any confusion caused. On the advice of the reviewer we moved one figure from the supplement to the main paper. Therefore the numbering of figures in the supplement changed between the first and the revised manuscript and we failed to capture all of these changes. There are four specific points raised by the reviewer here:

1. Incorrect legend for Figure 1 - We have corrected the error in the legend for Figure 1 (i.e changed "Figure S1" to "Figure 2").

2. Reply to point 4 in initial review - The reviewer did not refer to Figure S2 in point 4 of their previous review (they did refer to this in point 7). We do not know which question the reviewer invites us to reply to.

3. No label for phylogenetic tree in supplement - We have inserted a label for Figure S1.

4. Figure $\mathbf{S} 2$ labelled as Figure S3 - We have corrected this error.

Severe technical shortcomings remain and these are again outlined:

1. It is not sufficiently clarified how the analysis results in such an enormous loss of reads: Previously of 21.5 million reads only 400,493 were analyses, now it reads that from 11.7 million reads 606,227 could be analysed. Why were now fewer reads available? Why are less than $10 \%$ analysed? This is a very unusual yield for 165 sequencing. The authors refer to "high stringency" and p. 7 for further explanations in their response but this stringency is nowhere explained. I am afraid that important information is missed.

Our response: There are actually now more reads and a smaller proportion of "lost" reads included in the analysis reported in the revised manuscript $(606,227$ compared to 400,493$)$. There is no standard for stringency and, as the reviewer will be aware, the advantage of a higher cut off reduces the potential for "false positive" findings. In this study, we used the default settings in the workbench software (Qiagen). This has a very "high stringency" cut off which means that a lot of reads are removed. Our data are publicly available and colleagues can apply different stringency thresholds to our data. We have expanded the text on page 7 to explain our approach to stringency which explains the threshold used. Finally (as suggested by the reviewer) we have used a mock community to replicate data from our pipeline using second pipeline which applied a different stringency; this last analysis indicates that our stringency has not affected the diversity of microbiome detected but has increased the accuracy of detection (data now included in Table S2).

2. On "p.29 of 62" the authors again show the phylogenetic tree that should show "all analysed samples". In this tree I do neither see the genus Streptococcus which should be found in all healthy individuals at least in the mouth and throat or Haemophilus which has been reported as a typical proteobacterium in asthma patients. I come to the same conclusion as in (1.) that important 
information might be missing. That is why I requested to analyze a defined mock community but the authors refuse to do so. I do not see why this should not be possible even if the study was closed.

Our response: This phylogenetic tree shows details of phyla and does not provide details of species. Therefore species such as Streptococcus and Haemophilus are not shown. The analysis of mock community indicates that our pipeline more sensitive than a second pipeline and that important information is not missing. We have provided more labels on the phylogenetic tree identifying more phyla.

3. I previously stated that I am afraid that in bronchial samples the bacterial yield might have been low and therefore prone to contamination. Indeed the new Fig. 2 clearly shows that in the one control sample proteobacteria were brought in. I am afraid that in samples with known low yield (bronchial washing from healthy individuals) this might induce an error as previously reported (Salter et al. BMC Biology 2014, 12:87). I therefore requested to do an analysis of bacterial load (e.g. quantitative 16S PCR). The authors now refer me to Table S2 but this is entirely misleading. "Number of reads" has nothing to do with the original bacterial load. During the pipeline of mixing and barcoding for the sequencing process typically equal amounts of PCR products are taken. After the sequencing the number of reads only reflects variation in the sequencing and does not relate to the original bacterial load. Without seeing true data on bacterial load the issue of contamination cannot be discussed in sufficient depth.

Our response: We believe that the mock community analysis described previously addresses the issue of low DNA yield in bronchial samples (see Table S2). There was not sufficient sample volume to determine the bacterial yield. The mock community analysis and consistency with published data assure us that low bacterial load in bronchial samples has not substantially affected our results. We have added text acknowledging that we did not calculate DNA yield. We have also added a very recently published paper which also finds different clustering of bacterial communities in the upper and lower airways.

4. From Table $S 2$ it is visible that for the bronchial samples a number of samples had to be excluded because that did not give enough reads. I am afraid that the majority were from healthy children whereas those from asthmatic were analysed. The latter are known to have a higher Proteobacteria load. Therefore it is mandatory, to show the disease status in Fig. 2. Moreover, I would like to see separate PERMANOVAs for diseased and healthy children with respect to differences between sputa and bronchial washings. This is not covered so far by any of the analysis and from the Fig. on " $p .30$ of 62" (mislabeled as S3) it becomes clear that the difference between NBR and NSP is much less as compared to YBR VS. YSP. I do NOT want to make a conclusion on asthma patients but I state that the mixing of healthy and diseased people in on population might be a significant problem. This might be also visible when Fig. 2 would be labeled with the disease status: From a look at Fig. 2 it seems that at least 3-4 samples are much more similar to the throat/sputum. The conclusion of difference between BR and SP is mostly based on the unassigned sequences and for those the data do not sufficiently exclude a contamination issue (see my comment 3 ).

Our response: The reviewer makes a number of points here:

- Samples need labelling by asthma status in figure 2 . We have done this

- Separate PERMANOVA for children with and without asthma. We have done this (table S4)

- Difference between BR and SP is mostly based on unassigned sequences. We have now removed unassigned sequences from this figure and the majority of bronchial samples are predominantly the light blue colour corresponding to Proteobacteriae whereas the sputum samples have very few Proteobacteriae seen but are dominated by Fusobacteria and 
Bacteroidites. We do acknowledge that two bronchial samples (P12BR and P16 BR) have few Proteobacteriae and have a similar pattern to their corresponding sputum sample. When all patients are considered, there are significant differences between bronchial and sputum samples but we have modified the conclusions to acknowledge that in a minority sputum samples may be a valid index of bronchial samples.

5. Technically it remains unclear why in Fig. 1 unassigned sequences are shown but in Fig. 2 excluded. Moreover, on visual inspection there seems to be a discrepancy between proteobacteria abundance in Fig. 2 for all BR samples (for many samples $>50 \%$ ) as compared to Fig. 1 (20\%). How has the data in Fig. 1 been calculated (mean?). All in all the data do not sufficiently justify the main conclusion.

Our response: We have revised Figure 1 and removed the unassigned phyla. The reviewer is correct and data in Figure 1 are mean values. The proportion of proteobacteria in figure 2 is certainly $>50 \%$ for most individuals but this figure shows how two individuals (P12BR and p16BR) have very few proteobacteria and results from these two individuals reduce the mean proportion of proteobacteria to the value represented in Figure 1. We have amended the main conclusion (see point 4 above). 
Microbiome Characteristics of Induced Sputum compared to Bronchial Fluid and Upper

$$
\text { Airway Samples }
$$

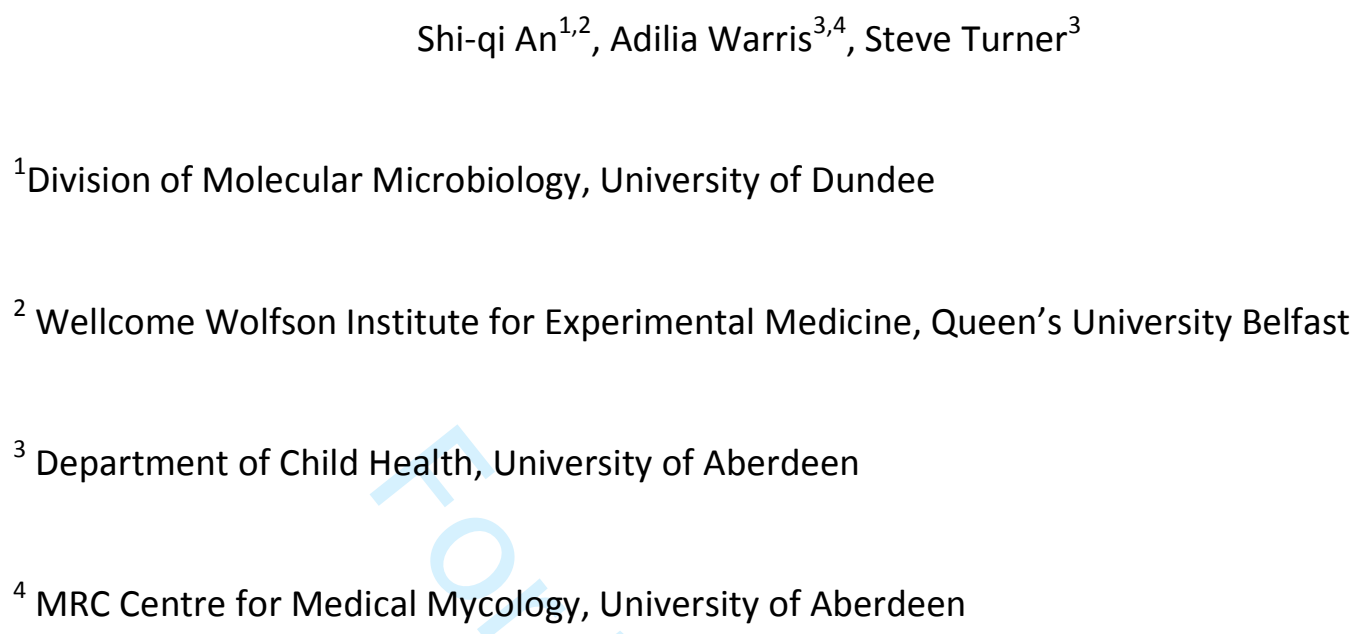

${ }^{1}$ Division of Molecular Microbiology, University of Dundee

${ }^{2}$ Wellcome Wolfson Institute for Experimental Medicine, Queen's University Belfast

${ }^{3}$ Department of Child Health, University of Aberdeen

${ }^{4}$ MRC Centre for Medical Mycology, University of Aberdeen

Contact details: Dr Steve Turner, Child Health, Royal Aberdeen Children's Hospital, Aberdeen, UK, AB25 2ZG. Tel +44 1224 438470. s.w.turner@abdn.ac.uk

Running head: Microbiota of induced sputum

Key words: Bronchial fluid, Child, Microbiota, Sputum 


\section{ABSTRACT}

Objective. The study of the community of microorganisms (the microbiota) in the lower airways in children is restricted to opportunistic sampling in children undergoing elective general anaesthetic. Here we tested the hypothesis that induced sputum is a valid alternative to directly sampling the lower airways to study lower airway microbiota.

Methods. Children scheduled for elective operations were recruited. Pre-operatively a sample of induced sputum was obtained. After anaesthesia was induced, a bronchial brushing and swabs of the upper respiratory tract were obtained. Bacterial community analysis was performed by amplification of the V3-V4 16S rRNA gene region.

Results. Twenty children were recruited, mean age 10.7 years. Induced sputum şamples were obtained from induced sputum in 12 children, bronchial brushing fromin 14 and $;$ nä of bacterial communities was similar in the mouth, throat and sputum samples with the nose and bronchial samples being different. Actinobacteria species dominated the nose and mouth, Fusobacteria were the dominant species in the throat and sputum whilst Proteobacteria species dominated in bronchial samples. Forty-one percent of detected bacteria in bronchial samples were unclassified. Bacterial communities from the mouth, throat and induced sputum were tightly clustered and were distinct from nose and those found in bronchial communities.

Conclusions. Induced sputum may not be a valid surrogate for microbiome assessment of the lower airways in all individuals. A large minority of Many bacteria in bronchial samples were not recognised by standard testing, suggesting that our understanding of the lower airway microbiota in children remains rudimentary. 


\section{INTRODUCTION}

The lower airways were traditionally considered sterile in healthy individuals, and this paradigm was based on observations that standard microbiology culture from samples collected from healthy individuals yielded no pathogens ${ }^{1}$. The advent of polymerase chain reaction (PCR) testing for bacterial DNA has now identified communities of bacteria in lower airway secretions from healthy individuals ${ }^{2,3}$. The relevance of bacteria in airway microbiota to respiratory symptoms is unclear, but there is evidence suggesting that the microbiota may be relevant to influence respiratory exacerbations $s^{2,4}$ or even to the aetiology of chronic respiratory conditions such as asthma ${ }^{5}$. Exacerbations of chronic conditions such as cystic fibrosis (CF) and chronic obstructive pulmonary disease are linked to a change ${ }^{2}$ (termed "airway dysbiosis") or reduction ${ }^{4}$ in the dominant bacterial species in the microbiota. In young infants, those carrying Haemophilus influenzae or Moraxella catarrhalis in the hypopharynx were more likely to have asthma symptoms at three years of age ${ }^{5}$.

A major challenge to studying the lower airway microbiota is obtaining samples without contamination from the upper airways ${ }^{3}$, and it is known that the microbiota in the naso- and oropharynx differ from that in the bronchus in adults and children ${ }^{6-8}$. Three papers have used bronchoscopic sampling to compare describe-the upper and lower airway microbiome in children and-whilst there are similarities in the dominant phyla identified in some studies, there are differences in genera identified and also in the clustering of bacterial communities $^{6-8}$. The characteristics of the lower airway microbiome may also vary between individuals depending on their asthma status ${ }^{6}$ and using a nasal or oral approach to the lower airways $^{8}$._reported a greater abundance of Proteobacteria species in those with 
asthma compared to controls ${ }^{6,7}$. Two studies in young people with CF have compared the microbiota in induced sputum and oropharyngeal swabs ${ }^{9,10}$, and both concluded that a throat swab sample may be a valid surrogate for sputum for microbiota analysis. Induced sputum is a non-invasive alternative to direct bronchial sampling, and is used in the clinical management of tuberculosis and CF in children and also in research. The study of the lower airway microbiome would be considerably easier if induced sputum was a valid surrogate of bronchial fluid. The aim of the current study was to collect induced sputum and samples from the bronchus, nose, throat and mouth in children and undertake a comprehensive assessment of upper and lower airway microbiota in children. Our hypothesis was that induced sputum is a valid alternative to directly sampling the lower airways to study lower airway microbiota.

\section{METHODS}

\section{Study design}

Children aged 5-16 years and scheduled for elective ear nose and throat operations at Royal Aberdeen Children's Hospital between January and June 2015 were invited to take part. All children were scheduled for tonsillectomy and some also had grommets inserted. These operations require endotracheal intubation, which allows sampling of the lower airways without contamination by the upper airways. Children younger than 5 years were excluded since they were unlikely to provide a sample of induced sputum. Other exclusion criteria included receipt of antibiotics within the last month and CF. In the hours before the operation (when the child was fasted), children took part in an assessment which included completion of a questionnaire, height and weight measurement, spirometry (in accordance 
with international guidelines ${ }^{11}$ ) and induction of sputum using $4 \%$ and if required $5 \%$ saline as previously described ${ }^{12}$. An adequate quality sputum sample was defined as the presence of a white sputum plug on a visual inspection; samples were not sufficiently large enough to allow for cell count analysis. Immediately after anaesthesia was induced and the endotracheal tube in situ, a sterile $2.7 \mathrm{~mm}$ interdental brush (Dento Care Professional, London UK) was used to collect a sample of nasal secretions and a separate brush interdental brush was gently brushed on the buccal surface of the mouth to collect oral secretions. A standard bacterial swab (Transwab, Medical Wire Equipment, Corsham, UK) was used to obtain a sample of pharyngeal secretions under direct inspection. Finally, a bronchoscopy cytology brush (10-mm disposable cytology brush, BC 202D-2010, Olympus, Southend-on-Sea, Essex, UK) was passed down the endotracheal tube until resistance was met and then rotated and withdrawn to obtain "blind" bronchial samples. Blood was taken for serum total IgE assay. The study was approved by the North of Scotland Research Ethics Committee (13/NS/0144), written parental consent was obtained and verbal assent was obtained from all participants.

\section{DNA extraction and sequencing}

The bacterial community analysis was carried out as described previously ${ }^{13}$ with some modification as detailed below. Briefly, samples taken from the nose, mouth, throat, sputum and bronchial brushing were frozen at $-80^{\circ} \mathrm{C}$ prior to analysis. Samples were then transported from Aberdeen to Dundee for processing. Upon arrival the sample had thawed and were suspended in phosphate buffer saline where genomic DNA was extracted and purified using the DNA/RNA All Prep kit $^{14}$ (Qiagen) and stored according to manufacturer's 
instructions. Standard protocol, 16S Metagenomic Sequencing Library Preparation Guide (Illumina), was followed to prepare sequencing libraries targeting the variable V3 and V4 regions of the 16S rRNA gene and paired-end sequencing was performed on the MiSeq System (Illumina). We followed sampling and controls procedures described by the Earlham Institute

(http://www.earlham.ac.uk/sites/default/files/docs/Genomic\%20Services/Sample\%20Guide lines\%20Aug17.pdf) and Illumina (https://support.illumina.com/documents/documentation/chemistry documentation/16s/1 6s-metagenomic-library-prep-guide-15044223-b.pdf which are designed to minimize the risk for contamination. Quality controls and sequencing were performed at Earlham Institute (Norwich, UK).

\section{Data and Statistical analysis}

Bioinformatics analyses on raw data was performed using the Biomedical Genomics Workbench version 4.0 (Qiagen) equipped with the Microbial Genomics Module version 2.0 (Qiagen) plugin. Sequences were imported and processed for optional merge paired reads, adapter trimming, fixed length trimming and then the sequences were filtered based on the number of reads to obtain sequences that are comparable in length and coverage for clustering. Quality and chimera filtering were performed using the recommended programme parameters [for complete details see www.qiagenbioinformatics.com]. Samples with low coverage were removed from further analysis. Operational Taxonomic Units (OTU) clustering and taxonomic assignment were done using Greengenes v13_5 (97\%) as reference. New OTUs were indicated when similarity percentage was lower than $80 \%$ with minimum occurrence of 5 reads. Low abundant OTUs were discarded from further analyses 
(minimum combined abundance was set at 10). Summary of processed sequence data is described in Table S1. MUSCLE was used for OTUs alignment in order to reconstruct a maximum likelihood phylogeny with neighbor joining as construction method and Jukes Cantor as nucleotide substitution model. We compared community structures and diversity across patient cohorts to determine if inter-cohort differences in structure were seen. To achieve this a rarefaction sampling analysis was carried out using a standard methodology ${ }^{15}$. In order to minimise the risk for contamination, we sought to identify a high (i.e. conservative) number of sequences required to characterise bacteria from the samples determined from rarefaction. In the absence of a standard international stringency cut off the default settings in the workbench software (Qiagen) were applied; the default settings filters out many reads and generates fewer unassigned reads and is therefore highly stringent. These adjustments were assessed by analyzing a previously characterized mock community to confirm that the data handling was consistent with other studies (sequencing files of the project: ERP021973,https://www.ebi.ac.uk/metagenomics/projects/ERP021973/samples/ERS15889 32). Data generated in the study was assessed using the Qiagen software and also applied to EBI Metagenomics analysis pipeline V3.0 to verify results (acknowledging that fewer reads would remain in the analysis with Qiagen). The findings in the controls were taken into account during the analysis. Alpha diversity was calculated using number of OTUs. Beta diversity was obtained using D_0.5 UniFrac and represented as Principal Coordinate Analysis (PCoA). Robustness analysis was performed using PERMANOVA with UniFrac distances. All sequencing data have been deposited in NCBI Sequence Read Archive (SRA) database under the follow BioProject ID: PRJNA388557 
RESULTS

\section{Study Subjects}

Twenty patients recruited of which 8 were boys, 7 with diagnosed asthma and the mean (standard deviation) age was 10.7 (2.8) years. Descriptives of the children recruited are given in Ttable 1. Induced sputum was obtained from 12 children and samples of fluid from bronchus, throat mouth and nose were obtained in all children. Three control samples were collected.

\section{Sample quality control}

A total of $11,750,879$ PCR reads were were analysed, and 606,227 high quality reads were obtained. The resulting OTU table contained 1053 OTUs, where 532 OTUs were assigned based on the Greengenes v13_5 (clustered at 97\% similarity), and 521 OTUs were novel (see Table S1). These processing criteria allowed data from 74 samples from patients for further analyses. Of these 15 were from the nose, 16 from mouth, 17 from throat, 12 from sputum and 14 from bronchial samples (Table S2). Samples from all five sites were available in six children. The median read counts for the five sites were as follows: bronchus 4438; mouth 4400; throat 4868; nose 8927; and sputum 11250. The results from the mock community analysis using Qiagen and EBI Metagenomics analysis pipelines were highly consistent. $\underline{\text { However Qiagen software assigned phyla for all samples whereas the EBI Metagenomics did }}$ not assign phyla in $15 \%$ of samples (see Table S3). 


\section{Bacterial abundance}

The relative bacterial abundance in each sample cohort is presented in Figure- 1 . The overall profile of bacterial communities was largely similar in the mouth, throat and sputum samples with the nose and bronchial being different. All sample cohorts contained five major bacterial phyla: Actinobacteria, Bacteroidetes, Firmicutes, Fusobacteria and Proteobacteria. The three most abundant phyla of bacteria observed by analysis in nose, mouth, throat, bronchial and sputum were Actinobacteria, Fusobacteria and Bacteroidetes (seen in Figure 1). Actinobacteria dominated in the nose (73\% of all total species) and mouth (37\%) samples, Fusobacteria dominated in throat (31\%) and sputum (38\%) samples and Proteobacteria were dominant in bronchial samples (34\%). Both nasal and bronchial samples had more unclassified OTUs ( $12 \%$ and $41 \%$ respectively) than other samples. The relative abundance of each individual sample is shown in Figure 2. Figure S1 presents the phylogenetic tree of the microbial community in all analysed samples. In two of the control samples there was no identifiable bacteria DNA signal however some DNA signal was detected in the one of the three control and this was taken into account during analysis (See Figure 2).

\section{Comparison between complete community structures within samples from different sites}

The rarefaction analysis suggested that 2000 sequences would be sufficient for characterizing the majority of bacteria present in these communities. The numbers of reads were lowest in bacterial communities from mouth and bronchus (Figure 3; Table S2). A comparison of individual bacterial communities, using PERMANOVA principal coordinate 
analysis (PCoA), showed a distinct clustering by sample cohorts based roughly on where the sample was taken from. Bacterial communities in samples from the mouth, throat and induced sputum were tightly clustered together $(\operatorname{padj}=1)$ and were distinct from those of the nose and bronchus (padj<0.001), which independently clustered (Figure 4; Table 2): these clustering results were seen when children with asthma and without asthma were considered separately (Table S4), Table S토 demonstrates that clustering of bacterial communities was not evident between sites among subgroups stratified by asthma/not asthma or atopy/not atopy.

The bacterial communities in the five sites sampled did not differ between the children with and without asthma; there was evidence of some differences in bacterial abundance and diversity between groups (see supplement Figure S2). A comparison of the abundant phyla of bacteria identified in bronchial samples taken from asthma and non-asthma patients showed the profiles were similar, but the abundance of a signal for phyla was different (but did not reach significance). In bronchial samples from asthma patients, the abundant phyla identified was Proteobacteria while those samples taken from non-asthma patients showed a greater abundance for Fusobacteria.

\section{DISCUSSION}

This study was designed to determine whether induced sputum is a valid alternative to directly sampling of the lower airways to study airway microbiota in children. The main finding was that the characteristics of the microbiota in induced sputum and in bronchial samples were different. A second finding was that a minority of bacterial DNA in bronchial 
samples was unclassified. In two individuals (P12 and P16, see Figure 2) the proportion of phyla identified from the bronchial sample was similar to the sputum sample but for the remainder of participants there were clear differences in the proportions of different phyla from bronchial and sputum samples. Together these findings suggest that induced sputum is not a valid surrogate for direct bronchial sampling to study the lower airway microbiota in all children, but that there is still a pressing need to better understand the lower airway microbiota in children. Opportunistic bronchial sampling under clinically-indicated general anaesthetic will be requiredshould remain the gold standard for studying lower airway microbiome in children.

To our knowledge there are only three studies which describe-have compared the microbiota of upper and lower airway secretions in healthy children or children with asthma $\frac{6-8}{}$. A study where the majority of recruits were adults showed that the bacterial community in the oropharynx and bronchus were similar in healthy individuals ${ }^{6}$. whereas One a-solely paediatric study found very little overlap between the microbiota of the oropharynx and lower airways ${ }^{7}$ whilst a second paediatric study found similar predominant phyla in upper and lower airway samples but different genera ${ }^{8}$. Both paediatric studies found significant differences in the clustering of bacterial communities in the upper and lower airways ${ }^{7,8}$. The difference indominant -phyla in between-bronchial and throat samples is not consistent between studies but there is consistency for upper airway samplespreviously described ${ }^{6,7}$. For example, the microbiota of the upper airways is predominated by bacteria from the Firmicutes (e.g. Staphylcoccus and Streptococcus) and the Actinobacteria phyla (e.g. Corynebacteria) in our study and three others ${ }^{6-8}$. In contrast and in bronchial samples, bacterial species within the Proteobacteria phylum (including 
Moraxella and Haemophilus) predominated-in bronchial samples in our study and one

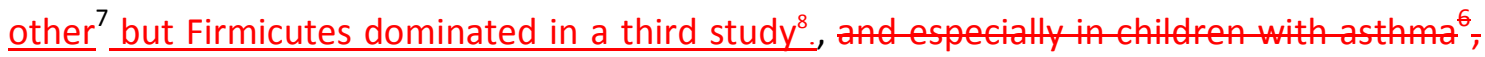
whereas-Differences in lower airway microbiome between studies may be due to different sampling methods ${ }^{8}$ and treatment with current or recent antibiotics ${ }^{7,8}$. in the microbiota of the upper airways is predominated by bacteria from the Firmicutes ${ }^{6,7}$ fe.g. Staphylcoccus and Streptococcus) and the Actinobacteria phyla ${ }^{6,7}$ (e.g. Corynebacteria).Our results, where we find no differences between the microbiome of induced sputum and throat swab, -are also consistent with previous studies which suggested that a throat swab may be a useful surrogate of induced sputum in young children with $\mathrm{CF}^{9,10}$. As our study did not include children with CF we cannot comment on the validity of induced sputum as an index of lower airway infection in this specific clinical setting. The consistency of our results with previous studies assures us that our findings are valid, despite the relatively small sample size.

Our study findings were based on samples taken from a group of children who were well. Recent work has given insight into the relationship between airway microbiota in the upper airways of children with acute infection, with results which differ from the present study. For example there is evidence that in the context of acute upper and lower respiratory tract infection, different bacterial species predominate in the nasopharynx (e.g. Proteobacteria and Firmicutes which contrast with Actinobacteria and Fusobacteria in our study ${ }^{15}$. The characteristics of the nasopharyngeal microbiota also change during the course of acute otitis media infection ${ }^{16}$.

Our study design minimised the risk of false positive results arising from contamination and from samples containing low DNA yields. There is no consensus on the number of control samples which should be collected for microbiome studies, and two of the three controls we 
collected contained no bacterial DNA and a third contained only bacteria with a very different profile of phyla compared to the samples from study subjects. We set out to have a high threshold for reads (>2000) and this filtered out samples with low DNA yields. Another source of potential contamination comes from reagents used to analyse the samples and this risk was minimised by taking standard precautions were taken and by including control samples in the analysis. Whilst we cannot exclude the possibility that some contamination may have occurred, our robust methodology and the consistency of our findings with previous studies assure us that any contamination has not substantially affected our findings.

Finding a small proportion of unassigned bacterial DNA in the nose and bronchial samples was unexpected and worthy of further investigation. The presence of "unclassified bacteria" has been described in nasopharyngeal samples from children ${ }^{7}$, but not in bronchial samples. We have carefully reviewed the unassigned sequences and removed human DNA sequences, and we therefore believe that there are a number of bacteria in the respiratory tract which are not identified by standard microbiota methodologies currently used.

We analysed data from a mock community (a "positive control") and this demonstrated that the Qiagen software used for analysis of our samples was able to detect bacteria in similar proportions to another software "pipeline". The Qiagen software identified all phyla whereas the alternative software could not assign $15 \%$ of bacterial DNA and this may be explained by the higher stringency set for the Qiagen software. There is not standard for $\underline{\text { stringency but these results suggest that although the higher stringency reduces the number }}$ of reads included in the analysis, the results are highly comparable to other methods and 
possibly less affected by potential contaminants. Our data are publicly available and colleagues are welcome to apply different stringencies to our data.

There are a number of settings where upper airway samples have been shown to be valid surrogates of lower airway samples for the clinical diagnosis of infective and non-infective conditions. Viral aetiology in bronchiolitis (a lower respiratory tract infection) can be established by nasopharyngeal aspirate ${ }^{16}$ and the nasal mucosa is used for diagnosis of primary ciliary dyskinesia ${ }^{17}$ and (in some situations) $\mathrm{CF}^{18}$. In the research setting, sampling from the upper airways has been validated as a surrogate for lower airway sampling ${ }^{19,20}$. However, we demonstrated that the microbiota of the nose was distinct to mouth and bronchus in most individuals and our results indicating that the microbiota of the upper airway is probably not a valid surrogate of lower airway microbiota in children, and Previous papers have suggested that with regards to lower airway samples, upper airway $\underline{\text { samples are "imperfect but reliable" }}$ and "both similar and different" ${ }^{8}$ and overall, our work finds more evidence for the upper airways samples being "imperfect and different" to lower airway samples and not "reliable and similar". has been shown by other as well.

The novelty of our study is that we demonstrate how the microbiota of induced sputum differs from bronchial samples and very closely matches the microbiota of mouth and throat, most likely due to contamination of lower airway secretions as they pass through the oropharynx, including sputum which is commonly mixed with expectorant. Our findings are consistent with a study of 78 children (mean age 2 years, almost half having received recent antibiotic treatment) which also describes differences in the microbiota between nose and mouth and between mouth and bronchial fluid ${ }^{7}$. Consistent with our findings, a study of adults and children (mean age 11 years) reports Actinobacteria being the predominant 
phyla in the nose but being almost absent from the oropharynx and bronchus. A different pattern is shown for Proteobacteria which are increasing from nose through to oropharynx and bronchus ${ }^{6}$. The apparent presence of different dominant bacterial communities in the upper and lower airways raises the question "where do bacteria in the lower airways originate?"

In children the lower airways are in direct communication with and adjacent to the oropharynx but there are several mechanisms that may lead to the establishment of different bacterial communities above and below the vocal cords. Perhaps most obviously, a child's mouth is regularly exposed to food and inedible items introduced to the oral cavity (including cutlery and fingers) whereas the lower airways are exposed only to inhaled exposures. Micro aspiration due to laryngopharyngeal reflux is thought to occur on a regular basis ${ }^{21}$ and exposure to acid and other gastric contents (including bacteria) may affect the microbiota of the lower airways but not the oropharynx. There are differences in the innate defences of the upper and lower airways which may also explain differences in their respective microbiota, for example lactoferrin concentrations are twice as high in the lower airways relative to upper airways ${ }^{22}$ whereas nitric oxide concentrations (known to have antimicrobial properties ${ }^{23}$ ) are typically one hundred times higher in the upper airways.

Our study was not designed to relate microbiota to clinical phenotypic data collected, for example asthma or atopy. However, in light of recent studies examining these parameters in children with asthma ${ }^{6,7}$ we made the best use of the data available, even with the limited number we had at our disposal. When examined the possible relationship between the bacteria present in children with and without asthma or atopy, we found no statistically 
differences between the bacteria when the samples were examined as a whole group or as specific sample region cohorts (nose, mouth, throat, bronchial and sputum samples), Figure S2 and Table S3. A descriptive analysis of the bronchial samples collected found Protoebacteria to be the dominant species and this is consistent with Hilty et al ${ }^{6}$.

There are some limitations to our study. First, the number of participants was relatively small. Second, induced sputum was not obtained in all participants and not all samples met our quality control criteria for bacterial DNA analysis and this resulted in missing data for some individuals. Third, as has been reported in other sputum microbiome studies ${ }^{6,7}$, we were not able to perform cell counts on the sputum pellet to determine the presence of neutrophils and absence of squamous epithelial cells. Finally, we did not prospectively calculate the DNA yield from samples although the consistency of our results with the small number of published studies $^{6,7}$, the consistency of results using both the Qiagen and EBI Metagenomics pipelines and the comparable results from mock communities assure is that low DNA yield has not altered the results. although we included control samples in our methodology we did not include samples from commercially available "mock" communities.

In summary, we report that neither induced sputum nor swabs from nose or mouth give an accurate indication of the bronchial microbiota in all children. For very obvious practical and ethical reasons, upper airway sampling will be necessary to study airway microbiome in many research setting and our findings adds to the evidence describing the limitations of this pragmatic approach. Our study was not designed to compare differences in the lower 
airway microbiota of children with and without asthma, and future research is required to supplement the little we do know about this potentially important subject.

\section{ACKNOWLEDGEMENTS}

The authors would like to thank the study participants and their parents. The study was supported by an NHS-Grampian Endowments. AW is supported by the Wellcome Trust Strategic Award (grant 097377) and the MRC Centre for Medical Mycology (grant MR/N006364/1) at the University of Aberdeen.

Competing interest statement. None of the authors has any competing interests. 


\section{REFERENCES}

1. Kahn FW, Jones JM. Diagnosing bacterial respiratory infection by bronchoalveolar lavage. J Infect Dis 1987; 155(5): 862-9.

2. Marsland BJ, Yadava K, Nicod LP. The airway microbiome and disease. Chest 2013; 144(2): 632-7.

3. Rogers GB, Shaw D, Marsh RL, Carroll MP, Serisier DJ, Bruce KD. Respiratory microbiota: addressing clinical questions, informing clinical practice. Thorax 2015; 70(1): 74-81.

4. Klepac-Ceraj V, Lemon KP, Martin TR, Allgier M, Kembel SW, Knapp AA, Lory S, Brodie EL, Lynch SV, et al. Relationship between cystic fibrosis respiratory tract bacterial communities and age, genotype, antibiotics and Pseudomonas aeruginosa. Environ Microbiol 2010; 12(5): 1293-303.

5. Bisgaard H, Hermansen MN, Bonnelykke K, Stokholm J, Baty F, Skytt NL, Aniscenko J, Kebadze T, Johnston SL. Association of bacteria and viruses with wheezy episodes in young children: prospective birth cohort study. BMJ 2010; 341: 4978.

6. Hilty M, Burke C, Pedro H, Cardenas P, Bush A, Bossley C, Davies J, Ervine A, Poulter L, Pachter L, et al. Disordered microbial communities in asthmatic airways. PLOS ONE 2010; 5(1): e8578.

7. Marsh RL, Kaestli M, Chang AB, Binks MJ, Pope CE, Hoffman LR, Smith-Vaughan HC. The microbiota in bronchoalveolar lavage from young children with chronic lung disease includes taxa present in both the oropharynx and nasopharynx. Microbiome 2016; 4: 37. 


\section{Kloepfer KM, Deschamp AR, Ross SE, Peterson-Carmichale SL, Hemmerich CM, Rusch DB, Davies SD. In children, the microbiota of the nasopharynx and bronchoalveolar lavage fluid are both similar and different. Ped Pulmonol 2018; DOI: 10.1002/ppul.23953}

9. Zemanick ET, Wagner BD, Robertson CE, Stevens MG, Szefler SJ, Accurso FJ, Sagel SD, Harris JK. Assessment of airway microbiota and inflammation in cystic fibrosis using multiple sampling methods. Ann Am Thor Soc 2015; 12(2): 221-9.

10. Boutin S, Graeber SY, Weitnauer M, Panitz J, StahI M, Clausznitzer D, Kaderali L, Einarsson G, Tunney MM, Elborn S, et al. Comparison of microbiomes from different niches of upper and lower airways in children and adolescents with cystic fibrosis. PLOS ONE 2015; 10(1): e0116029.

11. Miller MR, Hankinson J, Brusasco V, Burgos F, Casaburi R, Coates A, Crapo R, Enright P, van der Grinten CP, Gustaffson P, et al. Standardisation of spirometry. Eur Respir J 2005; 26(2): 319-38.

12. Pontin J, Blaylock MG, Walsh GM, Turner SW. Sputum eosinophil apoptotic rate is positively correlated to exhaled nitric oxide in children. Pediatr Pulmonol 2008; 43(11): 1130-4.

13. Twomey KB, Alston M, An S, O'Connell OJ, McCarthy Y, Swarbreck D, Febrer M, Dow JW, Plant BJ, Ryan RP. Microbiota and metabolite profiling reveal specific alterations in bacterial community structure and environment in the cystic fibrosis airway during exacerbation. PLOS ONE 2013; 8(12): e82432. 
14. Moen AEF, Tannaes TM, Vatn S, Ricanek P, Vatn MH, Jahnsen J, IBD-Character Consortium. Simultaneous purification of DNA and RNA from microbiota in a single colonic mucosal biopsy. BMC Research Notes 2016; 9: 328.

15. Zakharkina T, Martin-Loeches I, Matamoros S, Povoa P, Torres A, Kastelijn JB, Hofstra JJ, de Wever B, de Jong M, Schultz MJ, et al. The dynamics of the pulmonary microbiome during mechanical ventilation in the intensive care unit and the association with occurrence of pneumonia. Thorax 2017; 72: 803-10.

16. Kelly MS, Surette MG, Smieja M, Pernica JM, Rossi L, Lunistra, Steenhoff AP, Feemster KA, Goldfarb DM, Arscott-Mills T, Bioditswe S, Rulaganyang I et al. The Nasopharyngeal Microbiota of Children with Respiratory Infections in Botswana. Pediatr infect Dis J 2017;36:e211-e218

17. Chonmaitree T, Jennings K, Golovko G, Khanipov K, Pimenova M, Patel JA, McCormick DP, Loeffelholz MJ, Fofanov, Y. Nasopharyngeal microbiota in infants and changes during viral upper respiratory tract infection and acute otitis media. PLoS ONE 2017;12 (7):e0170630.

18. Ralston SL, Lieberthal AS, Meissner HC. Ralston SL, Lieberthal AS, Meissner HC, Alverson BK, Baley JE, Gadomski AM, Johnson DW, et al. Clinical Practice Guideline: The Diagnosis, Management, and Prevention of Bronchiolitis. Pediatrics. 2014;134(5):e1474e1502. Pediatrics 2015; 136(4): 782. 
19. Barbato A, Frischer T, Kuehni CE, Snijders D, Azevedo I, Bakati G, Bartoloni L, Escribano

A, Haarman E, Messelmar B, et al. Primary ciliary dyskinesia: a consensus statement on diagnostic and treatment approaches in children. Eur Respir J 2009; 34(6): 1264-76.

20. Farrell PM, Rosenstein BJ, White TB, Accurso FJ, Castellani C, Cutting GR, Durie PR, Legrys VA, Massie J, Parad RB, et al. Guidelines for diagnosis of cystic fibrosis in newborns through older adults: Cystic Fibrosis Foundation consensus report. J Pediatr 2008; 153(2): S4-S14.

21. Pringle EJ, Richardson HB, Miller D, Cornish DS, Devereux GS, Walsh GM, Turner SW. Nasal and bronchial airway epithelial cell mediator release in children. Pediatr Pulmonol 2012; 47(12): 1215-25.

22. Pitrez PMC, Brennan S, Turner S, Sly PD. Nasal wash as an alternative to bronchoalveolar lavage in detecting early pulmonary inflammation in children with cystic fibrosis. Respirology 2005; 10(2): 177-82.

23. Venkatesan NN, Pine HS, Underbrink M. Laryngopharyngeal reflux disease in children. Pediatr Clin North Am 2013; 60(4): 865-78.

24. Roca-Ferrer J, Mullol J, Perez M, Xaubet A, Momlins L, de Haro J, Shelhamer J, Picado C. Effects of topical glucocorticoids on in vitro lactoferrin glandular secretion: comparison between human upper and lower airways. J Allergy Clin Immunol 2000; 106(6): 1053-62.

24. Fang FC. Antimicrobial reactive oxygen and nitrogen species: concepts and controversies. Nature Rev Microbiol 2004; 2(10): 820-32. 
56

57

58

59

John Wiley \& Sons, Inc. 


\section{FIGURE LEGENDS}

Figure 1. Relative abundances of bacterial phyla identified as operational taxonomic units (OTUs) from the sequence reads generated from airway samples taken from children. The bar chart illustrates the taxonomic composition of each cohort of samples from a particular site. A detailed summary of the bacteria in each sample is described in Figure $\underline{2}\{1$.

Figure 2. Operational Taxonomic Units based relative sequence abundance of bacterial phyla based on 16S rRNA of all 75 samples. A detailed summary of the bacteria in each sample is described. On the horizontal axis, the number following $\mathrm{P}$ is the patient number and $\mathrm{NO}=$ nose, $\mathrm{MO}=$ mouth, $\mathrm{TH}=$ throat, $\mathrm{SP}=$ Sputum, $\mathrm{BR}=$ bronchial sample. $\mathrm{C} 2=$ the control sample where bacterial DNA was detected. $N / A=$ not applicable

Figure 3. Alpha diversity rarefaction curves of samples based on total number of observed Operational Taxonomic Units. Colour denotes different sample cohort ("nose" (NO), "mouth" (MO), "throat" (TH), "sputum" (SP) and "bronchial" (BR) samples).

Figure 4. Diversity analysis demonstrating differences in the bacterial phyla community between samples taken from airway samples taken from asthmatic and non-asthmatic children. Principal coordinate (PCo) analysis of all samples based on D_0.5 UniFrac distance. Colour denotes different sample cohort ("nose" (NO), "mouth" (MO), "throat" (TH), "sputum" (SP) and "bronchial" (BR) samples).

Figure S1. Phylogenetic tree of the microbial community in all analysed samples. All Operational Taxonomic Units are shown in this figure.

Figure S2. Bacterial phyla abundance and diversity between groups. On the horizontal axis, the first letter " $\mathrm{Y}$ " indicates that the child had asthma and " $\mathrm{N}$ " that they did not have 
asthma and $\mathrm{NO}=$ nose, $\mathrm{MO}=$ mouth, $\mathrm{TH}=$ throat, $\mathrm{SP}=$ Sputum, $\mathrm{BR}=$ bronchial sample. $\mathrm{N} / \mathrm{A}=$ not applicable

\section{TABLE LEGENDS}

Table 1. Details of the children recruited. SABA=short acting beta agonist, ICS=inhaled corticosteroids, $\mathrm{LABA}=$ long acting beta agonist

Table 2. PERMANOVA analysis results of testing differences in beta-diversity among different sample sites.

Table S1. Summary of sequence data used for Operational Taxonomic Units (OTU) clustering and analysis. The number of filtered reads and reads in OTUs doee not include the reads excluded by quality control

Table S2. List of sequence data for individual samples used for Operational Taxonomic Units (OTU) clustering and analysis.

\section{Table S3. Comparison of results from two pipelines used to analyse data. The Qiagen pipeline was used for the present study. The EBA pipeline (full name EBI Metagenomics analysis pipeline V3.0) was used as a comparator \\ Table S4. PERMANOVA analysis separately comparing beta-diversity among different sample sets for children with asthma and without asthma. \\ Table S5Table S3.PERMANOVA analysis comparing beta-diversity among different sample sets. Atopy was defined as serum IgE $>100 \mathrm{kU} / \mathrm{l}$.}


Whereas some minor issues have been addressed major issues are still not sufficiently replied to. I am still not convinced that one the main messages, namely that bronchial samples differ significantly from induced sputum, is justified by the data and therefore allows to conclude: "Induced sputum may not be a valid surrogate for microbiome assessment of the lower airways."

Before going into a deeper analysis I would like to mention that a considerable part of confusion comes due to erroneous labeling and numbering of the figures and tables. E.g. in my previous review I referred in point 4 to Fig. S2 which according to the legends was the phylogenetic tree (yet the Figure itself had no label) and claimed the absence of Streptococci and Haemophilus. In the reply the authors now refer to bar graphs that of course only show phyla. This is not a reply to my question. Moreover the current manuscript is still superficial in preparation: Fig. S2 as labeled in the legends ("Figure S2. Bacterial phyla abundance and diversity between groups") is sjown as Fig. S3 on p.30. In Fig. 1 the legend gives a note "A detailed summary of the bacteria in each sample is described in Figure S1." but this is now Fig. 2. Therefore I now always refer to the page number indicated at the top of the pdf file.

Our response: We apologise for any confusion caused. On the advice of the reviewer we moved one figure from the supplement to the main paper. Therefore the numbering of figures in the supplement changed between the first and the revised manuscript and we failed to capture all of these changes. There are four specific points raised by the reviewer here:

1. Incorrect legend for Figure 1 - We have corrected the error in the legend for Figure 1 (i.e changed "Figure S1" to "Figure 2").

2. Reply to point 4 in initial review - The reviewer did not refer to Figure $S 2$ in point 4 of their previous review (they did refer to this in point 7). We do not know which question the reviewer invites us to reply to.

3. No label for phylogenetic tree in supplement - We have inserted a label for Figure S1. The Pediatric Pulmonology web site does not allow for a legend to be inserted separately (in contrast with figures for the main paper where a legend is inserted separately).

4. Figure S2 labelled as Figure S3 - We have corrected this error.

\section{Severe technical shortcomings remain and these are again outlined:}

1. It is not sufficiently clarified how the analysis results in such an enormous loss of reads: Previously of 21.5 million reads only 400,493 were analyses, now it reads that from 11.7 million reads 606,227 could be analysed. Why were now fewer reads available? Why are less than $10 \%$ analysed? This is a very unusual yield for 165 sequencing. The authors refer to "high stringency" and p. 7 for further explanations in their response but this stringency is nowhere explained. I am afraid that important information is missed.

Our response: There are actually now more reads and a smaller proportion of "lost" reads included in the analysis reported in the revised manuscript $(606,227$ compared to 400,493$)$. There is no standard for stringency and, as the reviewer will be aware, the advantage of a higher cut off reduces the potential for "false positive" findings. In this study, we used the default settings in the workbench software (Qiagen). This has a very "high stringency" cut off which means that a lot of reads are removed. Our data are publicly available and colleagues can apply different stringency thresholds to our data. We have expanded the text on page 7 to explain our approach to stringency which explains the threshold used. Finally (as suggested by the reviewer) we have used a mock community to replicate data from our pipeline using second pipeline which applied a different stringency; this last analysis indicates that our stringency has not affected the diversity of microbiome detected but has increased the accuracy of detection (data now included in Table S3).

2. On "p.29 of 62" the authors again show the phylogenetic tree that should show "all analysed samples". In this tree I do neither see the genus Streptococcus which should be found in all healthy 
3. I previously stated that I am afraid that in bronchial samples the bacterial yield might have been low and therefore prone to contamination. Indeed the new Fig. 2 clearly shows that in the one control sample proteobacteria were brought in. I am afraid that in samples with known low yield (bronchial washing from healthy individuals) this might induce an error as previously reported (Salter et al. BMC Biology 2014, 12:87). I therefore requested to do an analysis of bacterial load (e.g. quantitative $16 S$ PCR). The authors now refer me to Table S2 but this is entirely misleading. "Number of reads" has nothing to do with the original bacterial load. During the pipeline of mixing and barcoding for the sequencing process typically equal amounts of PCR products are taken. After the sequencing the number of reads only reflects variation in the sequencing and does not relate to the original bacterial load. Without seeing true data on bacterial load the issue of contamination cannot be discussed in sufficient depth.

Our response: We believe that the mock community analysis described previously addresses the issue of low DNA yield in bronchial samples (see Table S3). There was not sufficient sample volume to determine the bacterial yield. The mock community analysis and consistency with published data assure us that low bacterial load in bronchial samples has not substantially affected our results. We have added text acknowledging that we did not calculate DNA yield. We have also added a very recently published paper which also finds different clustering of bacterial communities in the upper and lower airways.

4. From Table $S 2$ it is visible that for the bronchial samples a number of samples had to be excluded because that did not give enough reads. I am afraid that the majority were from healthy children whereas those from asthmatic were analysed. The latter are known to have a higher Proteobacteria load. Therefore it is mandatory, to show the disease status in Fig. 2. Moreover, I would like to see separate PERMANOVAs for diseased and healthy children with respect to differences between sputa and bronchial washings. This is not covered so far by any of the analysis and from the Fig. on " $p .30$ of 62" (mislabeled as S3) it becomes clear that the difference between NBR and NSP is much less as compared to YBR vs. YSP. I do NOT want to make a conclusion on asthma patients but I state that the mixing of healthy and diseased people in on population might be a significant problem. This might be also visible when Fig. 2 would be labeled with the disease status: From a look at Fig. 2 it seems that at least 3-4 samples are much more similar to the throat/sputum. The conclusion of difference between BR and SP is mostly based on the unassigned sequences and for those the data do not sufficiently exclude a contamination issue (see my comment 3 ).

Our response: The reviewer makes a number of points here:

- Samples need labelling by asthma status in figure 2. We have done this

- Separate PERMANOVA for children with and without asthma. We have done this (table S4)

- Difference between BR and SP is mostly based on unassigned sequences. We have now removed unassigned sequences from this figure and the majority of bronchial samples are 
predominantly the light blue colour corresponding to Proteobacteriae whereas the sputum samples have very few Proteobacteriae seen but are dominated by Fusobacteria and Bacteroidites. We do acknowledge that two bronchial samples (P12BR and P16 BR) have few Proteobacteriae and have a similar pattern to their corresponding sputum sample. When all patients are considered, there are significant differences between bronchial and sputum samples but we have modified the conclusions to acknowledge that in a minority sputum samples may be a valid index of bronchial samples.

5. Technically it remains unclear why in Fig. 1 unassigned sequences are shown but in Fig. 2 excluded. Moreover, on visual inspection there seems to be a discrepancy between proteobacteria abundance in Fig. 2 for all BR samples (for many samples $>50 \%$ ) as compared to Fig. 1 (20\%). How has the data in Fig. 1 been calculated (mean?). All in all the data do not sufficiently justify the main conclusion.

Our response: We have revised Figure 1 and removed the unassigned phyla. The reviewer is correct and data in Figure 1 are mean values. The proportion of proteobacteria in figure 2 is certainly $>50 \%$ for most individuals but this figure shows how two individuals (P12BR and p16BR) have very few proteobacteria and results from these two individuals reduce the mean proportion of proteobacteria to the value represented in Figure 1. We have amended the main conclusion (see point 4 above). 